Article

\title{
Cyanovinylation of Aldehydes: Organocatalytic Multicomponent Synthesis of Conjugated Cyanomethyl Vinyl Ethers
}

\author{
Samuel Delgado-Hernández ${ }^{1,2}\left(\mathbb{D}\right.$, Fernando García-Tellado ${ }^{1, *(\mathbb{D})}$ and David Tejedor ${ }^{1, *(\mathbb{D})}$ \\ 1 Instituto de Productos Naturales y Agrobiología, Consejo Superior de Investigaciones Científicas, \\ Astrofísico Francisco Sánchez 3, 38206 La Laguna, Spain; samuel050690@gmail.com \\ 2 Doctoral and Postgraduate School, Universidad de La Laguna, Apartado Postal 456, 38200 La Laguna, Spain \\ * Correspondence: fgarcia@ipna.csic.es (F.G.-T.); dtejedor@ipna.csic.es (D.T.)
}

\section{check for} updates

Citation: Delgado-Hernández, S.; García-Tellado, F.; Tejedor, D.

Cyanovinylation of Aldehydes: Organocatalytic Multicomponent

Synthesis of Conjugated

Cyanomethyl Vinyl Ethers. Molecules 2021, 26, 4120. https://doi.org/

$10.3390 /$ molecules 26144120

Academic Editors: Ana Maria

Gomez Neo and Carlos

Fernández Marcos

Received: 21 June 2021

Accepted: 2 July 2021

Published: 6 July 2021

Publisher's Note: MDPI stays neutral with regard to jurisdictional claims in published maps and institutional affiliations.

Copyright: (c) 2021 by the authors. Licensee MDPI, Basel, Switzerland. This article is an open access article distributed under the terms and conditions of the Creative Commons Attribution (CC BY) license (https:/ / creativecommons.org/licenses/by/ $4.0 /)$.
Abstract: A novel organocatalytic multicomponent cyanovinylation of aldehydes was designed for the synthesis of conjugated cyanomethyl vinyl ethers. The reaction was implemented for the synthesis of a 3-substituted 3-(cyanomethoxy)acrylates, using aldehydes as substrates, acetone cyanohydrin as the cyanide anion source, and methyl propiolate as the source of the vinyl component. The multicomponent reaction is catalyzed by $\mathrm{N}$-methyl morpholine $(2.5 \mathrm{~mol} \%)$ to deliver the 3-(cyanomethoxy)acrylates in excellent yields and with preponderance of the $E$-isomer. The multicomponent reaction manifold is highly tolerant to the structure and composition of the aldehyde (aliphatic, aromatic, heteroaromatics), and it is instrumentally simple (one batch, open atmospheres), economic ( $2.5 \mathrm{~mol} \%$ catalyst, stoichiometric reagents), environmentally friendly (no toxic waste), and sustainable (easy scalability).

Keywords: cyanomethyl vinyl ethers; 3-(cyanomethoxy)acrylates; multicomponent; cyanohydrin; cyanovinylation; organocatalysis; tertiary amine; $N$-methyl morpholine

\section{Introduction}

Cyanomethyl vinyl ethers (CMVEs) 1 constitute densely functionalized linear synthetic platforms which have found use as monomeric units in the construction of alkenyl ether-vinyl ester copolymers [1-5], as building blocks in the construction of multi-substituted 2,3-dihydrofurans [6,7] and 2,3-dihydropyrroles [7], and as convenient platforms for mechanistic investigations in the Claisen rearrangement of allyl vinyl ethers [8-10] (Scheme 1A). They are usually synthesized in a step-wise manner from the corresponding aldehydes through the formation of the $O$-formyl cyanohydrin intermediate and carbonyl methylenation [9] (Scheme 1B). When the vinyl moiety is endowed with electron withdrawing groups (e.g., 3), the cyanohydrin is directly converted into the conjugated cyanomethyl vinyl ether derivative by the amine-catalyzed Michael addition on the corresponding conjugated alkyne [6,7] (Scheme 1B). In both protocols, the corresponding cyanohydrin has to be synthesized and isolated to be used in the following reaction step. A direct synthesis of CMVEs 1 from the parent aldehydes should be desirable in terms of synthetic efficiency and both labor and step economies. With this idea in mind, we designed the three-component reaction (3CR) depicted in Scheme 1C, which implements a novel cyanovinylation of aldehydes to construct conjugated CMVEs 3 . To the best of our knowledge, this transformation has not been reported in the bibliography [11]. We report herein our results in the design and implementation of this $3 \mathrm{CR}$ and its practical application to the synthesis of methyl 3-substituted 3-(cyanomethoxy)acrylates (CMAs) 7. 
A) The cyanomethyl vinyl ether platform. A constellation of consonant functionalities.
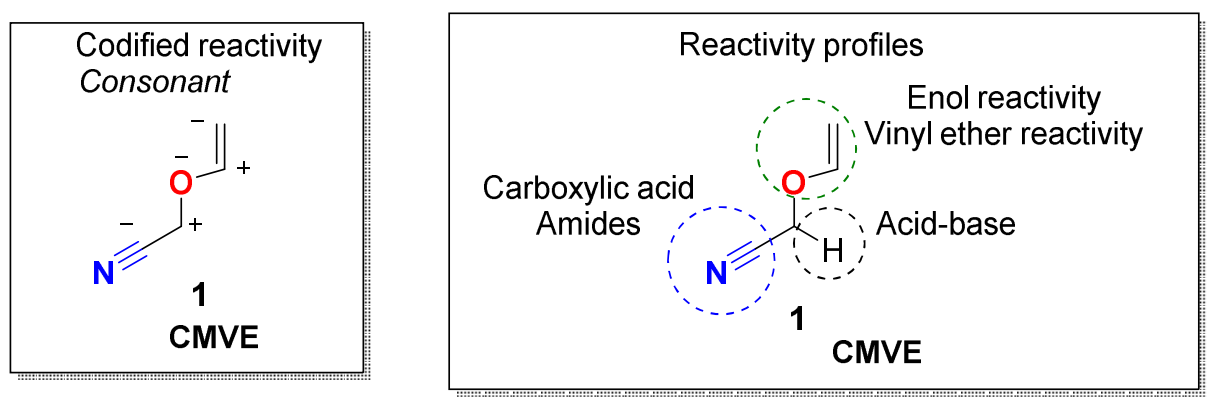

B) Synthesis of cyanomethyl vinyl ether platforms.

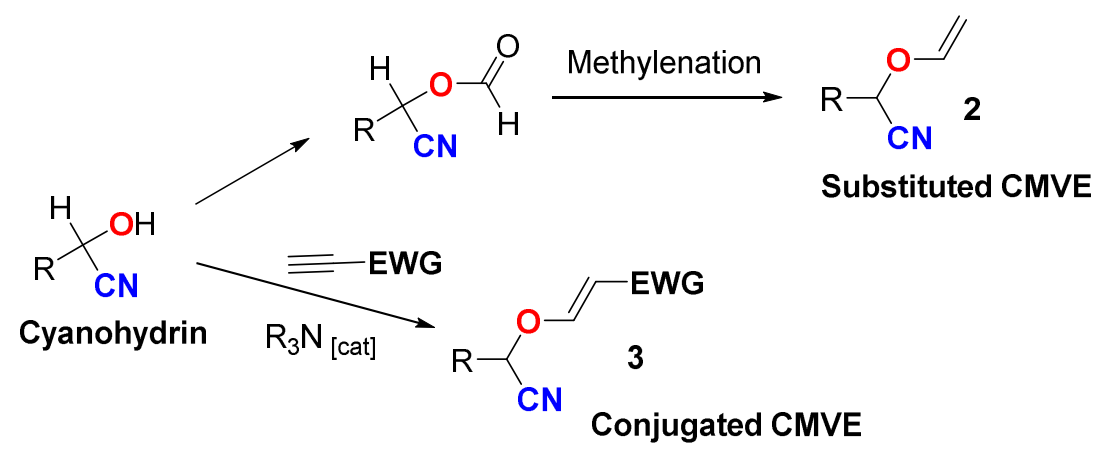

C) This work: multicomponent synthesis of conjugated cyanomethyl vinyl ether platforms.

$$
{ }^{-} \mathrm{C}=\mathrm{N}+\mathrm{RCHO}+\equiv \mathrm{EWG} \underset{\text { Cyanovinylation }}{\stackrel{\mathrm{R}_{3} \mathrm{~N}_{\text {[cat] }}}{\longrightarrow}} \mathrm{CN}_{\text {Conjugated CMVE }} \overbrace{}^{\mathrm{EWG}}
$$

Scheme 1. Cyanomethyl vinyl ethers platforms. (A) Properties, (B) previous synthesis, and (C) this work.

\section{Results and Discussion}

2.1. Design of a 3CR Manifold for the Synthesis of 3-(Cyanomethoxy)acrylates 7

The $3 \mathrm{CR}$ process was designed to operate under organocatalytic conditions according to the catalytic concept of "a good nucleophile generates a strong base" [12-15] (Scheme 2). This catalytic concept allows for launching base-driven processes by the in situ generation of catalytic amounts of a strong base in the reaction medium. It is technically performed by the reaction of a Lewis base (nucleophilic catalyst) on a conjugated alkyne to generate a conjugated vinyl anion (strong base). In the design of this 3CR manifold, we chose a tertiary amine as the Lewis base catalyst, because they have proved to be excellent catalysts for the in situ generation of allenolate anions type $\mathbf{I}[12,16]$. With this catalytic principle in mind, we designed the 3CR depicted in Scheme 2. The reaction manifold generates CMAs 7 through the catalytic cyanovinylation of an aldehyde substrate in the presence of stoichiometric amounts of cyanide anion and methyl propiolate (4). The choice of the cyanide anion source was an important design issue, because it had to be safe, environmentally friendly and suitable for the generation of cyanide anion under basic conditions, without introducing other reactive species into the catalytic cycle. Among of the commercially available cyanide precursors, we chose acetone cyanohydrin (5) because it meets all these criteria: it is safe [17] and it releases cyanide anion and acetone (waste) 
in the presence of bases [18]. The presence of acetone in the reaction medium should not introduce reactivity distortion issues in the catalytic process.

\section{A catalytic 3CR manifold for the cyanovinylation of aldehydes}

The catalytic concept

a good nucleophile generates a strong base

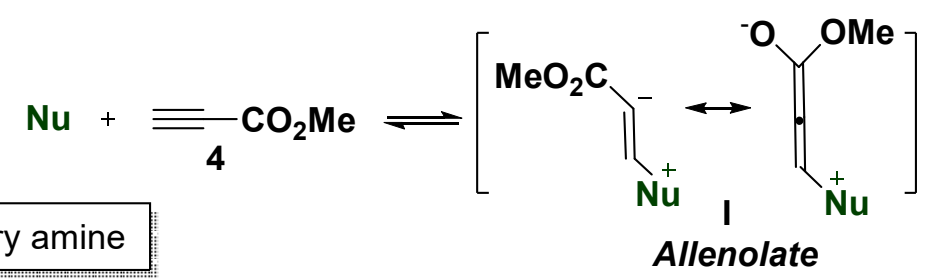

$$
\mathrm{Nu} \text { : tertiary amine }
$$

The catalytic 3CR manifold

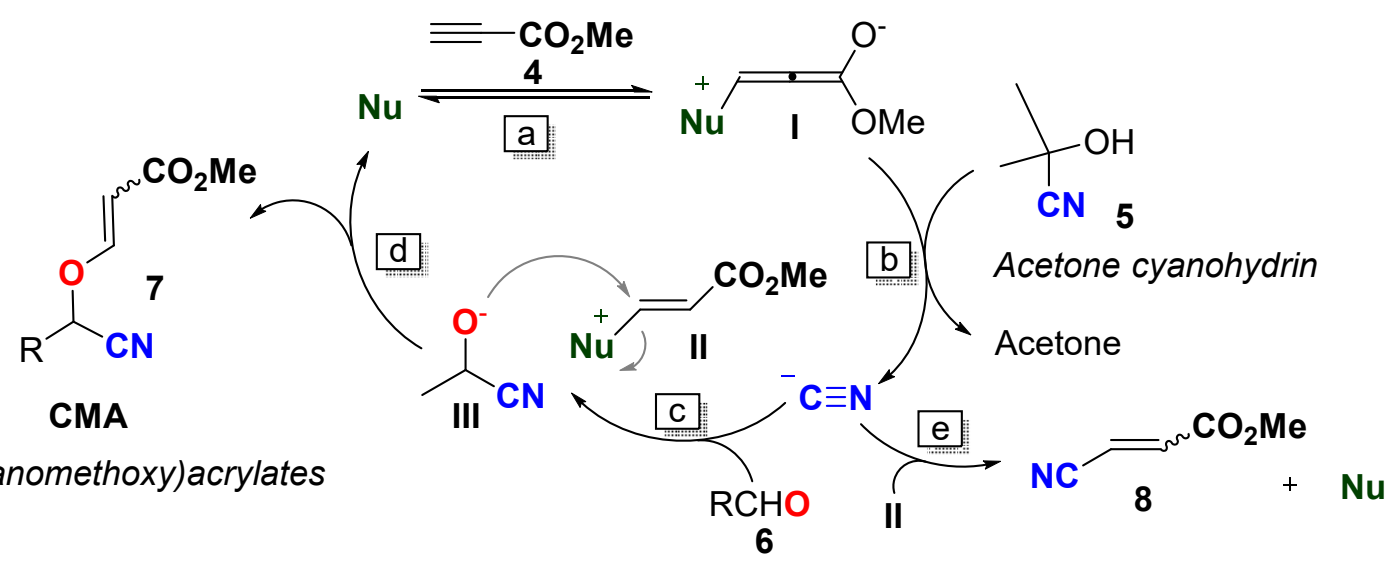

\section{Cycle reactions}

a Launching reaction: allenolate I formation.

b. Formation of cyanide/ammoniun acrylate II.

C Cycle propagating reaction.

d] Cycle closing reaction.

e Undesirable closing reaction.

\section{Manifold reactivity principles}

- The cyanohydrin III must be more reactive than the acetone cyanohydrin (5).

* The reaction of cyanide with the aldehyde (reaction c) has to be more favored than the conjugated addition on the amminum acrylate II to give the 3-cyanoacrylate 8 (reaction e).

Scheme 2. Catalytic 3CR for the synthesis of conjugated 3-(cyanomethoxy)acrylates 7.

With all considerations in mind, we envisioned the 3CR depicted in Scheme 2, which would be launched by the addition of the catalyst on the conjugated alkyne to generate the allenolate I (Scheme 2, reaction a). Allenolate I is a strong base, and it would deprotonate the acetone cyanohydrin (5) to generate the cyanide anion and the reactive ammonium acrylate II (reaction b). Addition of the cyanide anion on the aldehyde 6 would form the cyanohydrin III (reaction c), which would then add onto the ammonium acrylate II to deliver the final CMA 7 with liberation of the catalyst to reinitiate the cycle (reaction d). Based on our own experience [12-14,16], the catalytic cycle should deliver 7 as an E/Z mixture of isomers, with a clear preponderance of the $E$-isomer.

A critical concern in this design arises from the reactivity of the cyanide anion toward the aldehyde 6 versus the ammonium acrylate II (step c). The catalytic cycle requires that the cyanide anion fully react with the aldehyde to avoid the undesirable formation of methyl 3-cyanoacrylate 8 (reaction e) [12]. It must be noted that the formation of methyl 3cyanoacrylate $\mathbf{8}$ is also catalytic: its formation releases the catalyst to reinitiate the cycle [12]. 
Gratifyingly, the reactivity of the cyanide anion toward the aldehyde was good enough to mostly funnel the catalytic manifold toward formation of the CMA 7.

\subsection{Implementation of the $3 C R$ Manifold}

We chose the reaction of benzaldehyde (6a), acetone cyanohydrin (5) and methyl propiolate (4) as the benchmark reaction to find a set of convenient reaction conditions for the 3CR (Table 1). A wide panel of different tertiary amines and solvents was used in this exploratory study. Among all the combinations of tertiary amines and solvents assayed (entries 1-18), the combination of $N$-methylmorpholine (NMM) and $n$-hexanes showed to be the best, funneling the $3 \mathrm{CR}$ toward a nearly quantitative production of CMA 7a (99\%, E/Z:3/1) (only traces of 3-cyanoacrylate 8 were observed) (entry 8). More importantly, the NMM charge could be reduced up to $2.5 \mathrm{~mol} \%$ without significant loss in efficiency $(94 \%)$ and stereoselectivity $(2.5 / 1)$ (entry 21$)$. The effect of temperature on the stereoselectivity was studied carrying out the reaction at $0{ }^{\circ} \mathrm{C}$ and $-78{ }^{\circ} \mathrm{C}$, without observing any appreciable improvement at both temperatures (entries 22 and 23). As expected, no reaction was observed in the absence of catalyst (entry 24). Thus, we chose the conditions depicted in entry 21 as the standard reaction conditions: $N$-methyl morpholine $(2.5 \mathrm{~mol} \%), n$-hexanes, room temperature.

Table 1. Optimization of the 3CR manifold ${ }^{\text {a }}$.

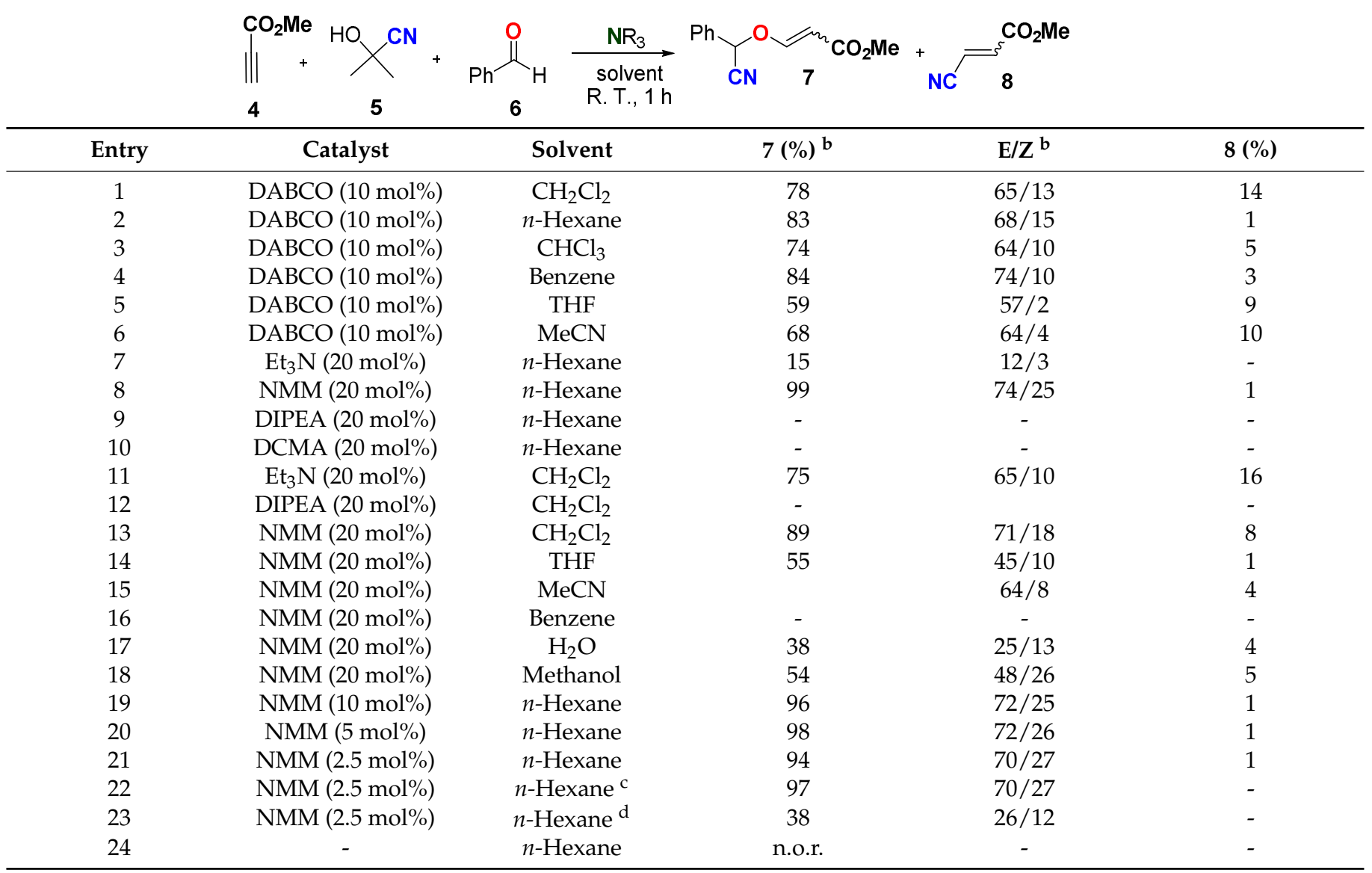

a Experimental conditions: benzaldehyde (6a) $(2 \mathrm{mmol})$, acetone cyanohydrin (5) (2 mmol), methyl propiolate (4) (2 mmol), $N$-methylmorpholine $(0.05 \mathrm{mmol}), n$-hexanes $(6 \mathrm{~mL}), \mathrm{R}$. T., $1 \mathrm{~h}$. ${ }^{\mathrm{b}}$ Yields and $E / Z$ ratios were calculated by ${ }^{1} \mathrm{H}$ NMR of crude reaction mixtures. ${ }^{\mathrm{c}} 0^{\circ} \mathrm{C}$. ${ }^{\mathrm{d}}-78^{\circ} \mathrm{C}$. DABCO $=1,4$-diazabicyclo[2.2.2] octane. NMM $=N$-methylmorpholine. DIPEA = -diisopropylethylamine. $\mathrm{DCMA}=\mathrm{N}, \mathrm{N}$-dicyclohexylmethylamine. $\mathrm{THF}=$ tetrahydrofuran. n.o.r. $=$ no observed reaction. 


\subsection{Scope of the 3CR Manifold}

The scope of the catalytic 3CR was studied using a wide array of different aldehydes 6 and the standardized reaction conditions (Scheme 3). Aldehydes were chosen to span a wide reactivity profile, including aromatic $(\mathbf{6 a}-\mathbf{o})$ heteroaromatic $(\mathbf{6} \mathbf{p}-\mathbf{q})$ and aliphatic $(\mathbf{6 r}-\mathbf{w})$. The reaction process was widely tolerant with respect to the aldehyde nature delivering the corresponding CMAs $7 \mathbf{a}-\mathbf{w}$ in yields spanning from $55 \%(7 \mathbf{u})$ to $99 \%$ ( $7 \mathbf{b}, 7 \mathbf{g}$ and $7 \mathbf{j})$.
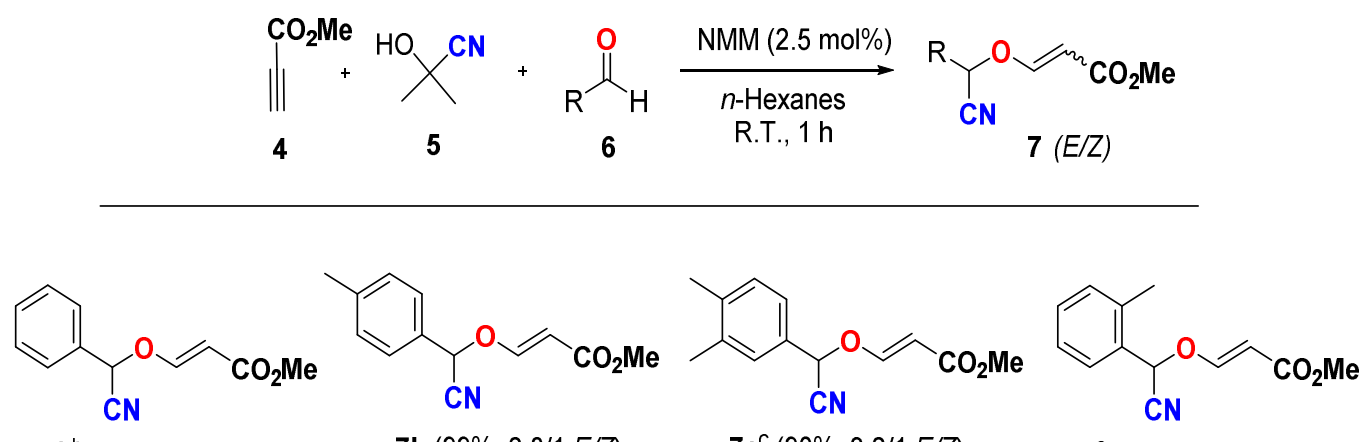

$7 \mathrm{a}^{\mathrm{a}, \mathrm{b}}(94 \% ; 2.5 / 1: E / Z)$

$7 \mathrm{~b}(99 \% ; 2.8 / 1: E / Z)$

$7 c^{\mathrm{C}}(90 \% ; 3.2 / 1: E / Z)$

$7 d^{\mathrm{C}}(96 \% ; 3.1 / 1: E / Z)$<smiles>CC(=O)/C=C/OC(C#N)c1c(C)cccc1C</smiles>

$7 \mathrm{e}^{\mathrm{c}}(91 \% ; 2.6 / 1: E / Z)$<smiles>COC(C)=O</smiles>

$7 f(91 \% ; 2.4 / 1: E / Z)$<smiles>COc1ccc(C(C#N)O/C=C/C(C)=O)c(OC)c1OC</smiles>

$7 g(99 \% ; 3.2 / 1: E / Z)$

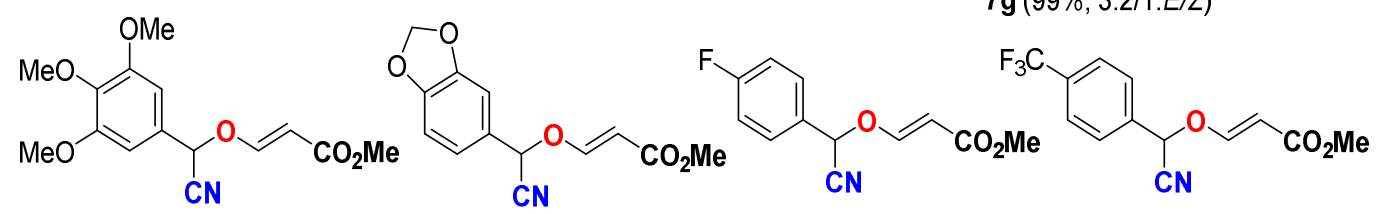

$7 \mathrm{~h}(92 \% ; 3.2 / 1: E / Z) \quad 7 \mathbf{i}(96 \% ; 2.6 / 1: E / Z)$

7j $(99 \% ; 2.8 / 1: E / Z)$

$7 \mathrm{k}(65 \% ; 3.2 / 1: E / Z)$

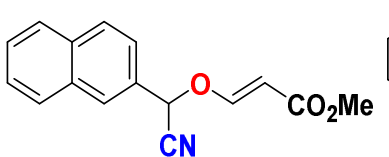

7I $(95 \% ; 2.5 / 1: E / Z)$<smiles>CC(=O)C=COC(C#N)c1cc2ccccc2c2ccccc12</smiles>

$70(97 \% ; 2.5 / 1: E / Z)$<smiles>CC(=O)C=COC(C#N)c1ccc2ccccc2c1C</smiles>

$7 \mathrm{~m}(73 \% ; 1.7 / 1: E / Z)$<smiles>CC(=O)/C=C/OC(C#N)c1cccs1</smiles>

7 p (90\%; 2.5/1:E/Z)<smiles>CC(=O)/C=C/OC(C#N)c1ccc2c(c1)Cc1ccccc1-2</smiles>

7n $(94 \% ; 2.5 / 1: E / Z)$

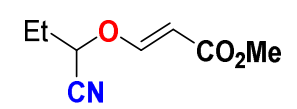

$\operatorname{7r}(91 \% ; 2.1 / 1: E / Z)$<smiles>CC(=O)C=COC(C#N)C(C)P</smiles>

7s $(90 \% ; 1.9 / 1: E / Z)$<smiles>CC(=O)C=COC(C#N)c1csc2ccccc12</smiles>

$7 q(92 \% ; 2.3 / 1: E / Z)$<smiles>CC(=O)C=COC(C#N)C(C)C</smiles>

$7 v(73 \% ; 2.1 / 1: E / Z)$<smiles>CCCCC(C#N)O/C=C/C(C)=O</smiles>
$7 \mathrm{t}(65 \% ; 1.9 / 1: E / Z)$<smiles>CC(=O)C=COC(C#N)C(C)(C)C</smiles>

$7 w^{C}(95 \% ; 2.5 / 1: E / Z)$

a Only the $E$-isomer is represented. ${ }^{b}$ The reaction was performed on a gram scale $(8 \mathrm{mmol} ; 91 \%$ yield, $2.4 / 1$ $E / Z) .{ }^{C} \mathrm{NMM}(12.5 \mathrm{~mol} \%)$, overnight.

Scheme 3. Aldehyde scope of the 3CR. 
Although the electronic nature of the aromatic rings did not show a general influence in the efficiency of the $3 \mathrm{CR}$ (compare $7 \mathbf{b}, 7 \mathrm{~g}$ and $7 \mathbf{j}$ ), the steric environment of the carbonyl function of the aldehyde was more determining, requiring, in the most steric-demanding cases, the use of higher charges of catalyst $(12.5 \mathrm{~mol} \%)$ and longer reaction times (overnight) to achieve good yields of CMAs (see $7 \mathbf{c}, 7 \mathbf{d}, 7 \mathbf{7 e}$ and $7 \mathbf{w}$ ). Under the standard reaction conditions, the hindered 1-methyl-2-naphthaldehyde (6m) and isobutanal (6v) delivered the corresponding $7 \mathrm{~m}$ and $7 \mathrm{v}$ in good yields $(73 \%)$, and their reactions were not further implemented. The reactivity of the linear aliphatic aldehydes was dependent on the length of the chain, being optimal for 2-3 carbon atoms $(7 \mathbf{r}, 7 \mathbf{s})$ and disadvantageous for longer chains $(7 \mathbf{t}, 7 \mathbf{u})$. With regard to the stereoselectivity of the reaction, the $E / Z$ ratio was moderate in all cases, spanning from $1.7 / 1$ for $7 \mathrm{~m}$ to $3.2 / 1$ for $7 \mathrm{c}, 7 \mathrm{~g}$, $7 \mathrm{~h}$ and $7 \mathrm{k}$.

Additionally, the scaling power of this $3 \mathrm{CR}$ and its suitability for real-world synthesis was established performing the reaction of benzaldehyde (6a) on a gram-scale $(8 \mathrm{mmol})$. Under these conditions, CMA 7a was obtained in $91 \%$ yield as a mixture of $E / Z$ isomers $(2.4 / 1)$.

Finally, and in accordance with our previous findings on the use of tertiary amines as catalysts for domino processes involving activated alkynes [13,16,19], the use of internal alkynes such as methyl 2-octynoate or ethyl phenylpropiolate did not lead to the formation of products.

\section{Materials and Methods}

\subsection{General Information}

${ }^{1} \mathrm{H}$ NMR and ${ }^{13} \mathrm{C}$ NMR spectra of $\mathrm{CDCl}_{3}$ solutions were recorded either at 400 and $100 \mathrm{MHz}$ or at 500 and $125 \mathrm{MHz}$ (Bruker Ac 200 and AMX2-500 respectively). ${ }^{1} \mathrm{H}$ and ${ }^{13} \mathrm{C}$ NMR spectra for compounds 7 are available from the Supplementary Materials. Highresolution mass spectra were recorded with a mass spectrometer LCT Premier XE (Manchester, UK) with two types of ionization sources: electrospray (ESI), an atmospheric pressure chemical ionization source (APCI), and with an orthogonal acceleration time-offlight (oaTOF) analyzer. Analytical thin-layer chromatography plates used were E. Merck Brinkman UV-active silica gel (Kieselgel 60 F254) on aluminum. Flash column chromatography was carried out with E. Merck silica gel 60 (particle size less than $0.020 \mathrm{~mm}$ ) using appropriate mixtures of ethyl acetate and hexanes, or ethyl acetate and dichloromethane as eluents. All reactions were performed in oven-dried glassware. All materials were obtained from commercial suppliers and used as received.

\subsection{General Procedure for the Synthesis of Cyanomethyl Vinyl Ether $7 a$}

To a solution of aldehyde (6) $(2.0 \mathrm{mmol})$, acetone cyanohydrin (5) $(2.0 \mathrm{mmol})$ and methyl propiolate $(4)(2.0 \mathrm{mmol})$ in $n$-hexanes $(6 \mathrm{~mL})$ was added $N$-methylmorpholine $(0.05 \mathrm{mmol})$ at once and the reaction mixture was stirred for $1 \mathrm{~h}$ at room temperature. The solvent was removed under reduced pressure, and the residue was purified by flash column chromatography (silica gel; $n$-hexane/ethyl acetate: $80 / 20$ ) to give the desired 3-(cyanomethoxy)acrylate 7.

\subsection{Characterization Data for 3-(cyanomethoxy)acrylates 7}

Methyl 3-(cyano(phenyl)methoxy)acrylate (7a). (E-isomer, major): (8.0 mol scale) (1.12 g, $64.5 \%)$. White solid: ${ }^{1} \mathrm{H}$ NMR $\left(\mathrm{CDCl}_{3}, 400 \mathrm{MHz}\right): \delta=3.71(\mathrm{~s}, 3 \mathrm{H}), 5.53(\mathrm{~d}, 1 \mathrm{H}, J=12.6 \mathrm{~Hz})$, $5.65(\mathrm{~s}, 1 \mathrm{H}), 7.46-7.53(\mathrm{~m}, 5 \mathrm{H}), 7.56(\mathrm{~d}, 1 \mathrm{H}, J=12.6 \mathrm{~Hz}) .{ }^{13} \mathrm{C} \mathrm{NMR}\left(\mathrm{CDCl}_{3}, 100 \mathrm{MHz}\right)$ : $\delta=51.5,70.3,101.3,115.2,127.4(2 \mathrm{C}), 129.4$ (2C), 130.7, 131.2, 158.1, 166.7 ppm. HRMS $\left(\mathrm{ESI}^{+}\right): m / z[\mathrm{M}+\mathrm{Na}]^{+}$calculated for $\mathrm{C}_{12} \mathrm{H}_{11} \mathrm{NO}_{3} \mathrm{Na} 240.0636$, found 240.0637. (Z-isomer, minor): (8.0 mol scale) (466 mg, 26.8\%). Yellowish solid: ${ }^{1} \mathrm{H}$ NMR $\left(\mathrm{CDCl}_{3}, 400 \mathrm{MHz}\right)$ : $\delta=3.69(\mathrm{~s}, 3 \mathrm{H}), 5.09(\mathrm{~d}, 1 \mathrm{H}, J=7.1 \mathrm{~Hz}), 5.83(\mathrm{~s}, 1 \mathrm{H}), 6.59(\mathrm{~d}, 1 \mathrm{H}, J=7.1 \mathrm{~Hz}), 7.45-7.46$ $(\mathrm{m}, 3 \mathrm{H}), 7.53-7.55(\mathrm{~m}, 2 \mathrm{H}) .{ }^{13} \mathrm{C} \mathrm{NMR}\left(\mathrm{CDCl}_{3}, 100 \mathrm{MHz}\right): \delta=51.2,71.9,101.0,115.6,127.3$ (2C), 129.4 (2C), 130.6, 131.3, 152.7, $164.5 \mathrm{ppm}$. 
Methyl 3-(cyano(p-tolyl)methoxy)acrylate (7b). (E-isomer, major): (341.2 mg, 74\%). White solid: ${ }^{1} \mathrm{H}$ NMR $\left(\mathrm{CDCl}_{3}, 400 \mathrm{MHz}\right): \delta=2.39(\mathrm{~s}, 3 \mathrm{H}), 3.71(\mathrm{~s}, 3 \mathrm{H}), 5.51(\mathrm{~d}, 1 \mathrm{H}, J=12.7 \mathrm{~Hz})$, $5.62(\mathrm{~s}, 1 \mathrm{H}), 7.27(\mathrm{~d}, 2 \mathrm{H}, J=8.1 \mathrm{~Hz}), 7.38(\mathrm{~d}, 2 \mathrm{H}, J=8.1 \mathrm{~Hz}), 7.54(\mathrm{~d}, 1 \mathrm{H}, J=12.7 \mathrm{~Hz}) .{ }^{13} \mathrm{C}$ $\operatorname{NMR}\left(\mathrm{CDCl}_{3}, 100 \mathrm{MHz}\right): \delta=21.2,51.3,70.2,101.1,114.8(2 \mathrm{C}), 115.4,127.4(2 \mathrm{C}), 128.2,130.0$ (2C), 141.0, 158.1, 166.7 ppm. HRMS $\left(\mathrm{ESI}^{+}\right): \mathrm{m} / z[\mathrm{M}+\mathrm{Na}]^{+}$calculated for $\mathrm{C}_{13} \mathrm{H}_{13} \mathrm{NO}_{3} \mathrm{Na}$ 254.0793, found 254.0791. (Z-isomer, minor): (120.0 mg, 26\%). Yellow oil: ${ }^{1} \mathrm{H}$ NMR $\left(\mathrm{CDCl}_{3}, 400 \mathrm{MHz}\right): \delta=2.32(\mathrm{~s}, 3 \mathrm{H}), 3.64(\mathrm{~s}, 3 \mathrm{H}), 5.01(\mathrm{~d}, 1 \mathrm{H}, J=7.2 \mathrm{~Hz}), 5.76(\mathrm{~s}, 1 \mathrm{H}), 6.53$ $(\mathrm{d}, 1 \mathrm{H}, J=7.2 \mathrm{~Hz}), 7.20(\mathrm{~d}, 2 \mathrm{H}, J=8.1 \mathrm{~Hz}), 7.37(\mathrm{~d}, 2 \mathrm{H}, J=8.1 \mathrm{~Hz}) .{ }^{13} \mathrm{C} \mathrm{NMR}\left(\mathrm{CDCl}_{3}\right.$, $100 \mathrm{MHz}): \delta=21.2,51.1,71.8,101.6,115.7$ (2C), $127.4(2 \mathrm{C}), 128.4,129.9(2 \mathrm{C}), 125.8,140.9$, $164.5 \mathrm{ppm}$.

Methyl 3-(cyano(3,4-dimethylphenyl)methoxy)acrylate (7c). (E-isomer, major): (337.0 mg, $69 \%)$. White solid: ${ }^{1} \mathrm{H}$ NMR $\left(\mathrm{CDCl}_{3}, 500 \mathrm{MHz}\right): \delta=2.25(\mathrm{~s}, 3 \mathrm{H}), 2.27(\mathrm{~s}, 3 \mathrm{H}), 3.67(\mathrm{~s}, 3 \mathrm{H})$, $5.47(\mathrm{~d}, 1 \mathrm{H}, \mathrm{J}=12.5 \mathrm{~Hz}), 5.55(\mathrm{~s}, 1 \mathrm{H}), 7.19(\mathrm{~s}, 2 \mathrm{H}), 7.23(\mathrm{~s}, 1 \mathrm{H}), 7.27(\mathrm{~d}, 2 \mathrm{H}, J=8.1 \mathrm{~Hz}), 7.38$ $(\mathrm{d}, 2 \mathrm{H}, J=8.1 \mathrm{~Hz}), 7.50(\mathrm{~d}, 1 \mathrm{H}, J=12.5 \mathrm{~Hz}) .{ }^{13} \mathrm{C} \mathrm{NMR}\left(\mathrm{CDCl}_{3}, 125 \mathrm{MHz}\right): \delta=19.6,19.7$, 51.4 $70.3,100.9,115.5,125.0,128.5,128.6,130.5,138.0,139.7$ 158.2, 166.9 ppm. HRMS $\left(\mathrm{ESI}^{+}\right)$: $m / z[\mathrm{M}+\mathrm{Na}]^{+}$calculated for $\mathrm{C}_{14} \mathrm{H}_{15} \mathrm{NO}_{3} \mathrm{Na} 268.0950$, found 268.0954. (Z-isomer, minor): (104.5 mg, 21\%). Transparent oil: ${ }^{1} \mathrm{H}$ NMR $\left(\mathrm{CDCl}_{3}, 500 \mathrm{MHz}\right): \delta=2.24(\mathrm{~s}, 3 \mathrm{H}), 2.25(\mathrm{~s}$, $3 \mathrm{H}), 3.65(\mathrm{~s}, 3 \mathrm{H}), 5.01(\mathrm{~d}, 1 \mathrm{H}, J=7.1 \mathrm{~Hz}), 5.74(\mathrm{~s}, 1 \mathrm{H}), 6.55(\mathrm{~d}, 1 \mathrm{H}, J=7.1 \mathrm{~Hz}), 7.17(\mathrm{~d}, 1 \mathrm{H}$,

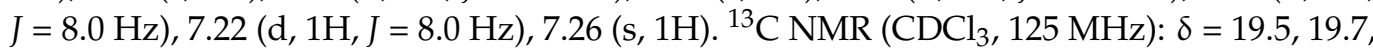
51.1, 71.8, 100.3, 115.8, 125.0, 128.5, 128.6, 130.4, 137.9, 139.6, 152.9, 164.7 ppm.

Methyl 3-(cyano(o-tolyl)methoxy)acrylate (7d). (E-isomer, major): (340.0 mg, 72\%). White solid: ${ }^{1} \mathrm{H}$ NMR $\left(\mathrm{CDCl}_{3}, 500 \mathrm{MHz}\right): \delta=2.40(\mathrm{~s}, 3 \mathrm{H}), 3.71(\mathrm{~s}, 3 \mathrm{H}), 5.55(\mathrm{~d}, 1 \mathrm{H}$, $\left.{ }^{3} J(\mathrm{H}, \mathrm{H})=12.4 \mathrm{~Hz}\right), 5.81(\mathrm{~s}, 1 \mathrm{H}), 7.26-7.32(\mathrm{~m}, 2 \mathrm{H}), 7.38(\mathrm{t}, 1 \mathrm{H}, J=7.5 \mathrm{~Hz}), 7.57(\mathrm{~d}, 1 \mathrm{H}$, $J=7.5 \mathrm{~Hz}), 7.59(\mathrm{~d}, 1 \mathrm{H}, J=12.4 \mathrm{~Hz}) .{ }^{13} \mathrm{C} \mathrm{NMR}\left(\mathrm{CDCl}_{3}, 125 \mathrm{MHz}\right): \delta=18.6,51.3,68.6,100.9$, 115.1, 126.7, 128.0, 129.1, 130.7, 131.4, 136.6, 158.0, 166.6 ppm. HRMS (ESI $\left.{ }^{+}\right): m / z$ [M + Na] ${ }^{+}$ calculated for $\mathrm{C}_{13} \mathrm{H}_{13} \mathrm{NO}_{3} \mathrm{Na} 254.0793$, found 254.0796. (Z-isomer, minor): (110.0 mg, 24\%). Transparent oil: ${ }^{1} \mathrm{H} \mathrm{NMR}\left(\mathrm{CDCl}_{3}, 500 \mathrm{MHz}\right): \delta=2.46(\mathrm{~s}, 3 \mathrm{H}), 3.68(\mathrm{~s}, 3 \mathrm{H}), 5.06(\mathrm{~d}, 1 \mathrm{H}$, $J=7.1 \mathrm{~Hz}), 5.88(\mathrm{~s}, 1 \mathrm{H}), 6.60(\mathrm{~d}, 1 \mathrm{H}, J=7.1 \mathrm{~Hz}), 7.25-7.30(\mathrm{~m}, 2 \mathrm{H}), 7.36(\mathrm{t}, 1 \mathrm{H}, J=7.5 \mathrm{~Hz})$, $7.59(\mathrm{~d}, 1 \mathrm{H}, J=7.5 \mathrm{~Hz}) .{ }^{13} \mathrm{C} \mathrm{NMR}\left(\mathrm{CDCl}_{3}, 125 \mathrm{MHz}\right): \delta=18.8,51.3,70.8,100.6,115.4,126.6$, $128.2,129.3,130.8,131.5,137.1,152.8,164.5$ ppm.

Methyl 3-(cyano(2,6-dimethylphenyl)methoxy)acrylate (7e) (E-isomer, major): (319.6 mg, 65\%). Transparent solid: ${ }^{1} \mathrm{H}$ NMR $\left(\mathrm{CDCl}_{3}, 400 \mathrm{MHz}\right): \delta=2.48(\mathrm{~s}, 6 \mathrm{H}), 3.70(\mathrm{~s}, 3 \mathrm{H}), 5.52$ $(\mathrm{d}, 1 \mathrm{H}, J=12.4 \mathrm{~Hz}), 5.97(\mathrm{~s}, 1 \mathrm{H}), 7.09(\mathrm{~d}, 2 \mathrm{H}, J=7.5 \mathrm{~Hz}), 7.23(\mathrm{t}, 1 \mathrm{H}, J=7.5 \mathrm{~Hz}), 7.50(\mathrm{~d}$, $1 \mathrm{H}, J=12.4 \mathrm{~Hz}) .{ }^{13} \mathrm{C} \mathrm{NMR}\left(\mathrm{CDCl}_{3}, 100 \mathrm{MHz}\right): \delta=20.0(2 \mathrm{C}), 51.1,65.8,100.7,115.1,127.8$, 129.6 (2C), 130.6, $137.6(2 \mathrm{C}), 158.2,166.8 \mathrm{ppm}$. HRMS $\left(\mathrm{ESI}^{+}\right): \mathrm{m} / z[\mathrm{M}+\mathrm{Na}]^{+}$calculated for $\mathrm{C}_{14} \mathrm{H}_{15} \mathrm{NO}_{3} \mathrm{Na} 268.0950$, found 268.0950. (Z-isomer, minor): (125.1 mg, 26\%). Transparent oil: ${ }^{1} \mathrm{H} \mathrm{NMR}\left(\mathrm{CDCl}_{3}, 500 \mathrm{MHz}\right): \delta=2.54(\mathrm{~s}, 6 \mathrm{H}), 3.69(\mathrm{~s}, 3 \mathrm{H}), 5.05(\mathrm{~d}, 1 \mathrm{H}, J=6.9 \mathrm{~Hz}), 6.00$ $(\mathrm{s}, 1 \mathrm{H}), 6.54(\mathrm{~d}, 1 \mathrm{H}, J=6.9 \mathrm{~Hz}), 7.08(\mathrm{~d}, 2 \mathrm{H}, J=7.6 \mathrm{~Hz}), 7.22(\mathrm{t}, 1 \mathrm{H}, J=7.6 \mathrm{~Hz}) .{ }^{13} \mathrm{C} \mathrm{NMR}$ $\left(\mathrm{CDCl}_{3}, 125 \mathrm{MHz}\right): \delta=20.0$ (2C), 51.1, 68.2, 100.2, 115.6, 128.1, $129.5(2 \mathrm{C}), 130.5,137.7$ (2C), 153.6, 164.5 ppm.

Methyl 3-(cyano(4-methoxyphenyl)methoxy)acrylate (7f) (E-isomer, major): (316.8 mg, $64 \%)$. White solid: ${ }^{1} \mathrm{H}$ NMR $\left(\mathrm{CDCl}_{3}, 400 \mathrm{MHz}\right): \delta=3.71(\mathrm{~s}, 3 \mathrm{H}), 3.81(\mathrm{~s}, 3 \mathrm{H}), 5.50(\mathrm{~d}$, $1 \mathrm{H}, J=12.6 \mathrm{~Hz}), 5.58(\mathrm{~s}, 1 \mathrm{H}), 6.97(\mathrm{~d}, 1 \mathrm{H}, J=8.7 \mathrm{~Hz}), 7.42(\mathrm{~d}, 1 \mathrm{H}, J=8.7 \mathrm{~Hz}), 7.52(\mathrm{~d}, 1 \mathrm{H}$, $J=12.6 \mathrm{~Hz}) .{ }^{13} \mathrm{C} \mathrm{NMR}\left(\mathrm{CDCl}_{3}, 100 \mathrm{MHz}\right): \delta=51.4,55.4,70.1,101.2,114.8(2 \mathrm{C}), 115.4$, $123.2,129.3$ (2C), 158.0, 161.5, 166.8 ppm. HRMS $\left(\mathrm{ESI}^{+}\right): \mathrm{m} / z[\mathrm{M}+\mathrm{Na}]^{+}$calculated for $\mathrm{C}_{13} \mathrm{H}_{13} \mathrm{NO}_{4} \mathrm{Na} 270.0742$, found 270.0743. (Z-isomer, minor): (130.9 mg, 27\%). Yellow solid: ${ }^{1} \mathrm{H} \mathrm{NMR}\left(\mathrm{CDCl}_{3}, 400 \mathrm{MHz}\right): \delta=3.68(\mathrm{~s}, 3 \mathrm{H}), 3.81(\mathrm{~s}, 3 \mathrm{H}), 5.05(\mathrm{~d}, 1 \mathrm{H}, J=7.1 \mathrm{~Hz}), 5.77(\mathrm{~s}$, $1 \mathrm{H}), 6.57(\mathrm{~d}, 1 \mathrm{H}, J=7.1 \mathrm{~Hz}), 6.94(\mathrm{~d}, 1 \mathrm{H}, J=8.7 \mathrm{~Hz}), 7.45(\mathrm{~d}, 1 \mathrm{H}, J=8.7 \mathrm{~Hz}) .{ }^{13} \mathrm{C} \mathrm{NMR}$ $\left(\mathrm{CDCl}_{3}, 100 \mathrm{MHz}\right): \delta=51.1,55.4,71.6,100.5,114.7(2 \mathrm{C}), 115.8,123.3,129.2(2 \mathrm{C}), 152.7,161.3$, $164.5 \mathrm{ppm}$.

Methyl 3-(cyano(2,3,4-trimethoxyphenyl)methoxy)acrylate (7g) (E-isomer, major): (467.1 mg, 76\%). Transparent oil: ${ }^{1} \mathrm{H}$ NMR $\left(\mathrm{CDCl}_{3}, 400 \mathrm{MHz}\right): \delta=3.65(\mathrm{~s}, 3 \mathrm{H}), 3.81(\mathrm{~s}, 3 \mathrm{H}), 3.84$ (s, 
3H), $3.92(\mathrm{~s}, 3 \mathrm{H}), 5.46(\mathrm{~d}, 1 \mathrm{H}, J=12.5 \mathrm{~Hz}), 5.88(\mathrm{~s}, 1 \mathrm{H}), 6.69(\mathrm{~d}, 1 \mathrm{H}, J=8.6 \mathrm{~Hz}), 7.21(\mathrm{~d}$, $1 \mathrm{H}, J=8.6 \mathrm{~Hz}), 7.52(\mathrm{~d}, 1 \mathrm{H}, J=12.5 \mathrm{~Hz}) .{ }^{13} \mathrm{C} \mathrm{NMR}\left(\mathrm{CDCl}_{3}, 100 \mathrm{MHz}\right): \delta=51.1,55.9,60.6$, $61.2,65.9,100.4,115.6,117.2,123.3,141.8,151.4,156.1,158.5,166.7$ ppm. HRMS $\left(\mathrm{ESI}^{+}\right)$: $m / z[\mathrm{M}+\mathrm{Na}]^{+}$calculated for $\mathrm{C}_{15} \mathrm{H}_{17} \mathrm{NO}_{6} \mathrm{Na} 330.0954$, found 330.0951. (Z-isomer, minor): (147.8 mg, 24\%). Yellow oil: ${ }^{1} \mathrm{H}$ NMR $\left(\mathrm{CDCl}_{3}, 400 \mathrm{MHz}\right): \delta=3.68(\mathrm{~s}, 3 \mathrm{H}), 3.86(\mathrm{~s}, 3 \mathrm{H}), 3.89$ $(\mathrm{s}, 3 \mathrm{H}), 3.98(\mathrm{~s}, 3 \mathrm{H}), 5.05(\mathrm{~d}, 1 \mathrm{H}, J=7.05 \mathrm{~Hz}), 5.97(\mathrm{~s}, 1 \mathrm{H}), 6.67(\mathrm{~d}, 1 \mathrm{H}, J=7.05 \mathrm{~Hz}), 6.73(\mathrm{~d}$, $1 \mathrm{H}, J=8.52 \mathrm{~Hz}), 7.32(\mathrm{~d}, 1 \mathrm{H}, J=8.52 \mathrm{~Hz}) .{ }^{13} \mathrm{C} \mathrm{NMR}\left(\mathrm{CDCl}_{3}, 100 \mathrm{MHz}\right): \delta=51.1,56.1,60.8$, $61.5,68.1,99.9,107.4,116.0,117.6,123.5,142.1,151.6,153.6,156.1,164.6$ ppm.

Methyl 3-(cyano(3,4,5-trimethoxyphenyl)methoxy)acrylate (7h) (E-isomer, major): (428.4 mg, $70 \%)$. Yellow oil: ${ }^{1} \mathrm{H} \mathrm{NMR}\left(\mathrm{CDCl}_{3}, 400 \mathrm{MHz}\right): \delta=3.67(\mathrm{~s}, 3 \mathrm{H}), 3.82(\mathrm{~s}, 3 \mathrm{H}), 3.84(\mathrm{~s}, 6 \mathrm{H}), 5.50$ $(\mathrm{d}, 1 \mathrm{H}, J=12.8 \mathrm{~Hz}), 5.60(\mathrm{~s}, 1 \mathrm{H}), 6.67(\mathrm{~s}, 2 \mathrm{H}), 7.51(\mathrm{~d}, 1 \mathrm{H}, J=12.8 \mathrm{~Hz}) .{ }^{13} \mathrm{C} \mathrm{NMR}\left(\mathrm{CDCl}_{3}\right.$, $100 \mathrm{MHz}): \delta=51.3,56.1(2 \mathrm{C}), 60.7,70.2,101.1,104.6(2 \mathrm{C}), 115.2,126.3,139.7,153.8(2 \mathrm{C})$, 157.9, 166.6 ppm. HRMS $\left(\mathrm{ESI}^{+}\right): \mathrm{m} / z[\mathrm{M}+\mathrm{Na}]^{+}$calculated for $\mathrm{C}_{15} \mathrm{H}_{17} \mathrm{NO}_{6} \mathrm{Na} 330.0954$, found 330.0951. (Z-isomer, minor): (135.1 mg, 22\%). Yellow solid: ${ }^{1} \mathrm{H} \mathrm{NMR}\left(\mathrm{CDCl}_{3}, 400\right.$ $\mathrm{MHz}): \delta=3.68(\mathrm{~s}, 3 \mathrm{H}), 3.83(\mathrm{~s}, 3 \mathrm{H}), 3.86(\mathrm{~s}, 6 \mathrm{H}), 5.10(\mathrm{~d}, 1 \mathrm{H}, J=7.1 \mathrm{~Hz}), 5.77(\mathrm{~s}, 1 \mathrm{H}), 6.58$ $(\mathrm{d}, 1 \mathrm{H}, J=7.1 \mathrm{~Hz}), 6.75(\mathrm{~s}, 2 \mathrm{H}) .{ }^{13} \mathrm{C} \mathrm{NMR}\left(\mathrm{CDCl}_{3}, 100 \mathrm{MHz}\right): \delta=51.1,56.2(2 \mathrm{C}), 60.7,71.9$, $100.9,104.5$ (2C), 115.6, 126.6, 139.6, 152.7 (2C), 153.9, 164.5 ppm.

Methyl 3-(benzo[d][1,3]dioxol-5-yl(cyano)methoxy)acrylate (7i). (E-isomer, major): (361.5 mg, 69\%). Orange solid: ${ }^{1} \mathrm{H}$ NMR $\left(\mathrm{CDCl}_{3}, 500 \mathrm{MHz}\right): \delta=3.70(\mathrm{~s}, 3 \mathrm{H}), 5.49(\mathrm{~d}, 1 \mathrm{H}, J=12.5 \mathrm{~Hz})$, $5.55(\mathrm{~s}, 1 \mathrm{H}), 6.01(\mathrm{~s}, 2 \mathrm{H}), 6.84(\mathrm{~d}, 1 \mathrm{H}, J=8.0 \mathrm{~Hz}), 6.94(\mathrm{~s}, 1 \mathrm{H}), 6.97(\mathrm{~d}, 1 \mathrm{H}, J=8.0 \mathrm{~Hz}), 7.50(\mathrm{~d}$, $1 \mathrm{H}, J=12.5 \mathrm{~Hz}) .{ }^{13} \mathrm{C} \mathrm{NMR}\left(\mathrm{CDCl}_{3}, 125 \mathrm{MHz}\right): \delta=51.4,70.1,101.2,101.9,107.7,108.7,115.3$, 122.0, 124.7, 148.6, 149.7, 157.9, $166.7 \mathrm{ppm}$. HRMS $\left(\mathrm{ESI}^{+}\right): \mathrm{m} / z[\mathrm{M}+\mathrm{Na}]^{+}$calculated for $\mathrm{C}_{13} \mathrm{H}_{11} \mathrm{NO}_{5} \mathrm{Na} 284.0535$, found 284.0530. (Z-isomer, minor): (140.7 mg, 27\%). Yellow solid: ${ }^{1} \mathrm{H} \mathrm{NMR}\left(\mathrm{CDCl}_{3}, 500 \mathrm{MHz}\right): \delta=3.69(\mathrm{~s}, 3 \mathrm{H}), 5.08(\mathrm{~d}, 1 \mathrm{H}, J=7.1 \mathrm{~Hz}), 5.72(\mathrm{~s}, 1 \mathrm{H}), 6.01(\mathrm{~s}$, $2 \mathrm{H}), 6.57(\mathrm{~d}, 1 \mathrm{H}, J=7.1 \mathrm{~Hz}), 6.84(\mathrm{~d}, 1 \mathrm{H}, J=8.0 \mathrm{~Hz}), 7.00(\mathrm{~s}, 1 \mathrm{H}), 7.02(\mathrm{~d}, 1 \mathrm{H}, J=8.0 \mathrm{~Hz}) .{ }^{13} \mathrm{C}$ NMR $\left(\mathrm{CDCl}_{3}, 125 \mathrm{MHz}\right): \delta=51.2,71.7,100.7,101.8,107.7,108.6,115.6,121.9,124.9,148.7$, 149.6, 152.6, $164.5 \mathrm{ppm}$.

Methyl 3-(cyano(4-fluorophenyl)methoxy)acrylate (7j) (E-isomer, major): (345.0 mg, 73\%). Transparent oil: ${ }^{1} \mathrm{H}$ NMR $\left(\mathrm{CDCl}_{3}, 500 \mathrm{MHz}\right): \delta=3.69(\mathrm{~s}, 3 \mathrm{H}), 5.12(\mathrm{~d}, 1 \mathrm{H}, J=12.5 \mathrm{~Hz}), 5.67$ $(\mathrm{s}, 1 \mathrm{H}), 7.14(\mathrm{t}, 2 \mathrm{H}, J=8.5 \mathrm{~Hz}), 7.48-7.51(\mathrm{~m}, 2 \mathrm{H}), 7.53(\mathrm{~d}, 1 \mathrm{H}, J=12.5 \mathrm{~Hz}) .{ }^{13} \mathrm{C} \mathrm{NMR}\left(\mathrm{CDCl}_{3}\right.$, $125 \mathrm{MHz}): \delta=51.5,69.4,101.3,112.2,116.5(\mathrm{~d}, 2 \mathrm{C}, J=22.6 \mathrm{~Hz}), 127.2(\mathrm{~d}, J=3.2 \mathrm{~Hz}), 129.6(\mathrm{~d}$, $2 \mathrm{C}, \mathrm{JCF}=8.9 \mathrm{~Hz}), 157.9,163.8(\mathrm{~d}, \mathrm{JCF}=250.8 \mathrm{~Hz}), 166.6 \mathrm{ppm} . \mathrm{HRMS}\left(\mathrm{ESI}^{+}\right): \mathrm{m} / z[\mathrm{M}+\mathrm{Na}]^{+}$ calculated for $\mathrm{C}_{12} \mathrm{H}_{10} \mathrm{FNO}_{3} \mathrm{Na} 258.0542$, found 258.0547. (Z-isomer, minor): (125.0 mg, 27\%). Yellow oil: ${ }^{1} \mathrm{H}$ NMR $\left(\mathrm{CDCl}_{3}, 500 \mathrm{MHz}\right): \delta=3.69(\mathrm{~s}, 3 \mathrm{H}), 5.12(\mathrm{~d}, 1 \mathrm{H}, J=7.1 \mathrm{~Hz})$, $5.81(\mathrm{~s}, 1 \mathrm{H}), 6.59(\mathrm{~d}, 1 \mathrm{H}, J=7.1 \mathrm{~Hz}), 7.14(\mathrm{t}, 2 \mathrm{H}, J=7.9 \mathrm{~Hz}), 7.55(\mathrm{t}, 2 \mathrm{H}, J=7.9 \mathrm{~Hz}) .{ }^{13} \mathrm{C}$ $\operatorname{NMR}\left(\mathrm{CDCl}_{3}, 125 \mathrm{MHz}\right): \delta=51.2,71.2,101.1,115.5,116.5(\mathrm{~d}, 2 \mathrm{C}, \mathrm{JCF}=22.2 \mathrm{~Hz}), 127.3$ (d, $J=2.8 \mathrm{~Hz}), 129.4(\mathrm{~d}, 2 \mathrm{C}, J=8.8 \mathrm{~Hz}), 152.7,163.8(\mathrm{~d}, J=251.0 \mathrm{~Hz}), 164.4 \mathrm{ppm}$.

Methyl 3-(cyano(4-(trifluoromethyl)phenyl)methoxy)acrylate (7k). (E-isomer, major): (283.2 mg, $50 \%)$. White solid: ${ }^{1} \mathrm{H} \mathrm{NMR}\left(\mathrm{CDCl}_{3}, 400 \mathrm{MHz}\right): \delta=3.72(\mathrm{~s}, 3 \mathrm{H}), 5.55(\mathrm{~d}, 1 \mathrm{H}, J=12.5 \mathrm{~Hz})$, $5.76(\mathrm{~s}, 1 \mathrm{H}), 7.57(\mathrm{~d}, 1 \mathrm{H}, J=12.5 \mathrm{~Hz}), 7.65(\mathrm{~d}, 2 \mathrm{H}, J=8.3 \mathrm{~Hz}), 7.74(\mathrm{~d}, 2 \mathrm{H}, J=8.3 \mathrm{~Hz}) .{ }^{13} \mathrm{C}$ $\operatorname{NMR}\left(\mathrm{CDCl}_{3}, 100 \mathrm{MHz}\right): \delta=51.5,69.3,101.7,114.7,123.4(\mathrm{q}, J=270.3 \mathrm{~Hz}), 126.4(\mathrm{q}, 2 \mathrm{C}$, $J=3.6 \mathrm{~Hz}), 127.7(2 \mathrm{C}), 132.8(\mathrm{q}, J=33.5 \mathrm{~Hz}), 134.9,157.8,166.5 \mathrm{ppm}$. HRMS $\left(\mathrm{ESI}^{+}\right): \mathrm{m} / z$ $[\mathrm{M}+\mathrm{Na}]^{+}$calculated for $\mathrm{C}_{13} \mathrm{H}_{10} \mathrm{~F}_{3} \mathrm{NO}_{3} \mathrm{Na}$ 308.0510, found 308.0510. (Z-isomer, minor): (88.0 mg, 15\%). Yellow solid: ${ }^{1} \mathrm{H}$ NMR $\left(\mathrm{CDCl}_{3}, 400 \mathrm{MHz}\right): \delta=3.71(\mathrm{~s}, 3 \mathrm{H}), 5.18(\mathrm{~d}, 1 \mathrm{H}$, $J=6.9 \mathrm{~Hz}), 5.89(\mathrm{~s}, 1 \mathrm{H}), 6.62(\mathrm{~d}, 1 \mathrm{H}, J=6.9 \mathrm{~Hz}), 7.73(\mathrm{dd}, 4 \mathrm{H}, J=8.6 \mathrm{~Hz}$ and $4.6 \mathrm{~Hz}) .{ }^{13} \mathrm{C}$ $\operatorname{NMR}\left(\mathrm{CDCl}_{3}, 100 \mathrm{MHz}\right): \delta=51.2,71.2,101.8,115.1,123.4(\mathrm{q}, J=273.8 \mathrm{~Hz}), 126.4(\mathrm{q}, 2 \mathrm{C}$, $J=3.9 \mathrm{~Hz}), 127.5(2 \mathrm{C}), 132.6(\mathrm{q}, J=33.1 \mathrm{~Hz}), 135.2,152.5,164.3 \mathrm{ppm}$.

Methyl 3-(cyano(naphthalen-2-yl)methoxy)acrylate (71). (E-isomer, major): (360.9 mg, 67\%). White solid: ${ }^{1} \mathrm{H}$ NMR $\left(\mathrm{CDCl}_{3}, 400 \mathrm{MHz}\right): \delta=3.71(\mathrm{~s}, 3 \mathrm{H}), 5.57(\mathrm{~d}, 1 \mathrm{H}, J=12.6 \mathrm{~Hz})$, $5.81(\mathrm{~s}, 1 \mathrm{H}), 7.56(\mathrm{~d}, 1 \mathrm{H}, J=8.6 \mathrm{~Hz}), 7.55-7.58(\mathrm{~m}, 2 \mathrm{H}), 7.60(\mathrm{~d}, 1 \mathrm{H}, J=12.6 \mathrm{~Hz}), 7.86-7.89(\mathrm{~m}$, $2 \mathrm{H}), 7.92(\mathrm{~d}, 1 \mathrm{H}, J=8.6 \mathrm{~Hz}), 7.99(\mathrm{~s}, 1 \mathrm{H}) .{ }^{13} \mathrm{C} \mathrm{NMR}\left(\mathrm{CDCl}_{3}, 100 \mathrm{MHz}\right): \delta=51.4,70.5,101.4$, $115.3,123.6,127.2,127.6,127.7,127.8,128.3,128.4,129.6,132.7,133.9,158.0,166.7$ ppm. 
HRMS (ESI $\left.{ }^{+}\right): m / z[\mathrm{M}+\mathrm{Na}]^{+}$calculated for $\mathrm{C}_{16} \mathrm{H}_{13} \mathrm{NO}_{3} \mathrm{Na} 290.0793$, found 290.0794. (Zisomer, minor): (147.6 mg, $28 \%)$. Yellow solid: ${ }^{1} \mathrm{H}$ NMR $\left(\mathrm{CDCl}_{3}, 400 \mathrm{MHz}\right): \delta=3.71(\mathrm{~s}, 3 \mathrm{H})$, $5.09(\mathrm{~d}, 1 \mathrm{H}, J=7.0 \mathrm{~Hz}), 6.00(\mathrm{~s}, 1 \mathrm{H}), 6.64(\mathrm{~d}, 1 \mathrm{H}, J=7.0 \mathrm{~Hz}), 7.55-7.58(\mathrm{~m}, 3 \mathrm{H}), 7.84-7.89(\mathrm{~m}$, $2 \mathrm{H}), 7.91(\mathrm{~d}, 1 \mathrm{H}, J=8.6 \mathrm{~Hz}), 8.05(\mathrm{~s}, 1 \mathrm{H}) .{ }^{13} \mathrm{C} \mathrm{NMR}\left(\mathrm{CDCl}_{3}, 100 \mathrm{MHz}\right): \delta=51.1,72.1,100.9$, $115.6,123.7,127.1,127.4,127.6,127.7,128.4,128.5,129.7,132.7,133.9,152.7,164.5$ ppm.

Methyl 3-(cyano(3-methylnaphthalen-2-yl)methoxy)acrylate (7m). (E-isomer, major): (233.8 mg, $46 \%)$. White solid: ${ }^{1} \mathrm{H}$ NMR $\left(\mathrm{CDCl}_{3}, 500 \mathrm{MHz}\right): \delta=2.66(\mathrm{~s}, 3 \mathrm{H}), 3.71(\mathrm{~s}, 3 \mathrm{H}), 5.58(\mathrm{~d}, 1 \mathrm{H}$, $J=12.4 \mathrm{~Hz}), 6.40(\mathrm{~s}, 1 \mathrm{H}), 7.33(\mathrm{~d}, 1 \mathrm{H}, J=8.4 \mathrm{~Hz}), 7.52(\mathrm{t}, 1 \mathrm{H}, J=7.9 \mathrm{~Hz}), 7.57(\mathrm{~d}, 1 \mathrm{H}$, $J=12.4 \mathrm{~Hz}), 7.62(\mathrm{t}, 1 \mathrm{H}, J=7.9 \mathrm{~Hz}), 7.87(\mathrm{~d}, 2 \mathrm{H}, J=8.4 \mathrm{~Hz}), 8.22(\mathrm{~d}, 1 \mathrm{H}, J=8.4 \mathrm{~Hz}) .{ }^{13} \mathrm{C}$ $\operatorname{NMR}\left(\mathrm{CDCl}_{3}, 125 \mathrm{MHz}\right): \delta=20.5,51.4,65.5,100.9,115.5,123.1,123.8,125.9,127.6,128.0$, 129.0, 130.6, 131.6, 132.8, 136.2, 158.2, $166.8 \mathrm{ppm}$. HRMS $\left(\mathrm{ESI}^{+}\right): \mathrm{m} / z[\mathrm{M}+\mathrm{Na}]^{+}$calculated for $\mathrm{C}_{17} \mathrm{H}_{15} \mathrm{NO}_{3} \mathrm{Na}$ 304.0950, found 304.0955. (Z-isomer, minor): (137.6 mg, 27\%). Brown solid: ${ }^{1} \mathrm{H} \mathrm{NMR}\left(\mathrm{CDCl}_{3}, 500 \mathrm{MHz}\right): \delta=2.70(\mathrm{~s}, 3 \mathrm{H}), 3.69(\mathrm{~s}, 3 \mathrm{H}), 5.03(\mathrm{~d}, 1 \mathrm{H}, J=7.0 \mathrm{~Hz}), 6.44$ $(\mathrm{s}, 1 \mathrm{H}), 6.56(\mathrm{~d}, 1 \mathrm{H}, J=7.0 \mathrm{~Hz}), 7.34(\mathrm{~d}, 1 \mathrm{H}, J=8.4 \mathrm{~Hz}), 7.52(\mathrm{t}, 1 \mathrm{H}, J=7.4 \mathrm{~Hz}), 7.64(\mathrm{t}, 1 \mathrm{H}$,

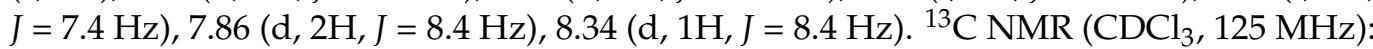
$\delta=20.5,51.3,67.7,100.8,116.0,123.4,124.1,125.9,127.7,128.9,129.1,130.8,131.6,132.9$, $136.6,153.2,164.6 \mathrm{ppm}$.

Methyl 3-(cyano(9H-fluoren-2-yl)methoxy)acrylate (7n). (E-isomer, major): (412.1 mg, $67 \%)$. Yellow solid: ${ }^{1} \mathrm{H}$ NMR $\left(\mathrm{CDCl}_{3}, 500 \mathrm{MHz}\right): \delta=3.72(\mathrm{~s}, 3 \mathrm{H}), 3.92(\mathrm{~s}, 2 \mathrm{H}), 5.55(\mathrm{~d}$, $1 \mathrm{H}, J=12.5 \mathrm{~Hz}), 5.70(\mathrm{~s}, 1 \mathrm{H}), 7.35-7.42(\mathrm{~m}, 2 \mathrm{H}), 7.49(\mathrm{~d}, 1 \mathrm{H}, J=7.5 \mathrm{~Hz}), 7.57(\mathrm{~d}, 1 \mathrm{H}$, $J=7.5 \mathrm{~Hz}), 7.59(\mathrm{~d}, 1 \mathrm{H}, J=12.5 \mathrm{~Hz}), 7.68(\mathrm{~s}, 1 \mathrm{H}), 7.80-7.84(\mathrm{~m}, 2 \mathrm{H}) .{ }^{13} \mathrm{C} \mathrm{NMR}\left(\mathrm{CDCl}_{3}\right.$, $125 \mathrm{MHz}$ ): $\delta=36.7,51.4,70.5,101.1,115.5,120.4,120.5,124.1,125.1,126.4,126.9,127.7$, 129.0, 140.1, 143.5, 144.1, 144.2, 158.2, $166.7 \mathrm{ppm}$. HRMS $\left(\mathrm{ESI}^{+}\right): \mathrm{m} / z[\mathrm{M}+\mathrm{Na}]^{+}$calculated for $\mathrm{C}_{19} \mathrm{H}_{15} \mathrm{NO}_{3} \mathrm{Na} 328.0950$, found 328.0951. (Z-isomer, minor): (162.2 mg, $27 \%$ ). Orange solid: ${ }^{1} \mathrm{H} \mathrm{NMR}\left(\mathrm{CDCl}_{3}, 500 \mathrm{MHz}\right): \delta=3.72(\mathrm{~s}, 3 \mathrm{H}), 3.94(\mathrm{~s}, 2 \mathrm{H}), 5.10(\mathrm{~d}, 1 \mathrm{H}, J=7.1 \mathrm{~Hz}), 5.90$ $(\mathrm{s}, 1 \mathrm{H}), 6.65(\mathrm{~d}, 1 \mathrm{H}, J=7.1 \mathrm{~Hz}), 7.35-7.42(\mathrm{~m}, 2 \mathrm{H}), 7.56(\mathrm{t}, 2 \mathrm{H}, J=7.6 \mathrm{~Hz}), 7.74(\mathrm{~s}, 1 \mathrm{H}), 7.81$ $(\mathrm{d}, 1 \mathrm{H}, J=7.6 \mathrm{~Hz}), 7.84(\mathrm{~d}, 1 \mathrm{H}, J=7.6 \mathrm{~Hz}) .{ }^{13} \mathrm{C} \mathrm{NMR}\left(\mathrm{CDCl}_{3}, 125 \mathrm{MHz}\right): \delta=36.9,51.2,72.2$, 100.8, 115.8, 120.4, 120.5, 124.2, 125.2, 126.4, 127.0, 127.8, 129.3, 140.3, 143.6, 144.2, 144.3, $152.8,164.6 \mathrm{ppm}$.

Methyl 3-(cyano(phenanthren-9-yl)methoxy)acrylate (7o). (E-isomer, major): (434.8 mg, $69 \%)$. White solid: ${ }^{1} \mathrm{H} \mathrm{NMR}\left(\mathrm{CDCl}_{3}, 500 \mathrm{MHz}\right): \delta=3.71(\mathrm{~s}, 3 \mathrm{H}), 5.54(\mathrm{~d}, 1 \mathrm{H}, J=12.5 \mathrm{~Hz})$, $6.25(\mathrm{~s}, 1 \mathrm{H}), 7.35-7.42(\mathrm{~m}, 4 \mathrm{H}), 7.66(\mathrm{~d}, 1 \mathrm{H}, J=12.5 \mathrm{~Hz}), 7.91-7.94(\mathrm{~m}, 2 \mathrm{H}), 8.07(\mathrm{~s}, 1 \mathrm{H}), 8.64$ $(\mathrm{d}, 1 \mathrm{H}, J=8.4 \mathrm{~Hz}), 8.71(\mathrm{~d}, 1 \mathrm{H}, J=8.4 \mathrm{~Hz}) .{ }^{13} \mathrm{C} \mathrm{NMR}\left(\mathrm{CDCl}_{3}, 125 \mathrm{MHz}\right): \delta=51.4,69.4$, 101.5, 115.1, 122.6, 123.3, 123.5, 124.6, 127.4, 127.5, 127.6, 127.8, 128.7, 129.4, 129.5, 129.9, 130.6, 131.3, 157.6, $166.7 \mathrm{ppm}$. HRMS $\left(\mathrm{ESI}^{+}\right): \mathrm{m} / z[\mathrm{M}+\mathrm{Na}]^{+}$calculated for $\mathrm{C}_{20} \mathrm{H}_{15} \mathrm{NO}_{3} \mathrm{Na}$ 340.0950, found 340.0946. (Z-isomer, minor): (177.2 mg, $28 \%$ ). White solid: ${ }^{1} \mathrm{H}$ NMR $\left(\mathrm{CDCl}_{3}, 500 \mathrm{MHz}\right): \delta=3.68(\mathrm{~s}, 3 \mathrm{H}), 5.03(\mathrm{~d}, 1 \mathrm{H}, J=7.1 \mathrm{~Hz}), 6.39(\mathrm{~s}, 1 \mathrm{H}), 6.68(\mathrm{~d}, 1 \mathrm{H}$, $J=7.1 \mathrm{~Hz}), 7.62(\mathrm{t}, 1 \mathrm{H}, J=6.9 \mathrm{~Hz}), 7.65-7.72(\mathrm{~m}, 3 \mathrm{H}), 7.90(\mathrm{~d}, 1 \mathrm{H}, J=7.9 \mathrm{~Hz}), 8.08(\mathrm{~s}, 1 \mathrm{H})$, $8.10(\mathrm{~d}, 1 \mathrm{H}, J=6.9 \mathrm{~Hz}), 8.60(\mathrm{~d}, 1 \mathrm{H}, J=8.4 \mathrm{~Hz}), 8.66(\mathrm{~d}, 1 \mathrm{H}, J=8.4 \mathrm{~Hz}) .{ }^{13} \mathrm{C} \mathrm{NMR}\left(\mathrm{CDCl}_{3}\right.$, $125 \mathrm{MHz}): \delta=51.1,71.1,100.8,115.5,122.5,123.3,123.8,124.8,127.3,127.5,127.6,127.8$, $128.6,129.4,129.8,129.8,130.8,131.3,151.9,164.4 \mathrm{ppm}$.

Methyl 3-(cyano(thiophen-2-yl)methoxy)acrylate (7p). (E-isomer, major): (282.0 mg, 64\%). Orange oil: ${ }^{1} \mathrm{H}$ NMR $\left(\mathrm{CDCl}_{3}, 400 \mathrm{MHz}\right): \delta=3.70(\mathrm{~s}, 3 \mathrm{H}), 5.52(\mathrm{~d}, 1 \mathrm{H}, J=12.6 \mathrm{~Hz}), 5.91(\mathrm{~s}$, $1 \mathrm{H}), 7.04(\mathrm{dd}, 1 \mathrm{H}, J=5.1$ and $3.6 \mathrm{~Hz}), 7.33(\mathrm{~d}, 1 \mathrm{H}, J=3.6 \mathrm{~Hz}), 7.47(\mathrm{~d}, 1 \mathrm{H}, J=5.1 \mathrm{~Hz}), 7.52$ $(\mathrm{d}, 1 \mathrm{H}, J=12.6 \mathrm{~Hz}) .{ }^{13} \mathrm{C} \mathrm{NMR}\left(\mathrm{CDCl}_{3}, 100 \mathrm{MHz}\right): \delta=51.4,65.5,101.6,114.6,127.3,129.4$, $129.5,132.9,157.3,166.6 \mathrm{ppm}$. HRMS $\left(\mathrm{ESI}^{+}\right): \mathrm{m} / z[\mathrm{M}+\mathrm{Na}]^{+}$calculated for $\mathrm{C}_{10} \mathrm{H}_{9} \mathrm{NO}_{3} \mathrm{SNa}$ 246.0201, found 246.0201. (Z-isomer, minor): (115.1 mg, $26 \%)$. Orange oil: ${ }^{1} \mathrm{H} ~ N M R\left(\mathrm{CDCl}_{3}\right.$, $400 \mathrm{MHz}): \delta=3.70(\mathrm{~s}, 3 \mathrm{H}), 5.11(\mathrm{~d}, 1 \mathrm{H}, J=7.2 \mathrm{~Hz}), 6.06(\mathrm{~s}, 1 \mathrm{H}), 6.63(\mathrm{~d}, 1 \mathrm{H}, J=7.2 \mathrm{~Hz})$, $7.06(\mathrm{dd}, 1 \mathrm{H}, J=5.1$ and $3.7 \mathrm{~Hz}), 7.38(\mathrm{~d}, 1 \mathrm{H}, J=3.7 \mathrm{~Hz}), 7.50(\mathrm{~d}, 1 \mathrm{H}, J=5.1 \mathrm{~Hz}) .{ }^{13} \mathrm{C} \mathrm{NMR}$ $\left(\mathrm{CDCl}_{3}, 100 \mathrm{MHz}\right): \delta=51.2,67.0,101.3,114.9,127.4,129.7,129.8,133.2,151.6,164.4 \mathrm{ppm}$.

Methyl 3-(benzo[b]thiophen-3-yl(cyano)methoxy)acrylate (7q). (E-isomer, major): (347.8 mg, $64 \%)$. Yellow solid: ${ }^{1} \mathrm{H}$ NMR $\left(\mathrm{CDCl}_{3}, 500 \mathrm{MHz}\right): \delta=3.71(\mathrm{~s}, 3 \mathrm{H}), 5.59(\mathrm{~d}, 1 \mathrm{H}, J=12.5 \mathrm{~Hz})$, 
$6.00(\mathrm{~s}, 1 \mathrm{H}), 7.43-7.49(\mathrm{~m}, 2 \mathrm{H}), 7.60(\mathrm{~d}, 1 \mathrm{H}, J=12.5 \mathrm{~Hz}), 7.80(\mathrm{~d}, 1 \mathrm{H}, J=7.2 \mathrm{~Hz}), 7.83(\mathrm{~s}$, $1 \mathrm{H}), 7.90(\mathrm{~d}, 1 \mathrm{H}, J=7.2 \mathrm{~Hz}),{ }^{13} \mathrm{C} \mathrm{NMR}\left(\mathrm{CDCl}_{3}, 125 \mathrm{MHz}\right): \delta=51.5,65.4,101.6,114.6,121.5$, $123.1,125.2,125.5,125.6,129.4,135.4,140.7,157.5,166.6$ ppm. HRMS $\left(\mathrm{ESI}^{+}\right): \mathrm{m} / z$ [M + Na] ${ }^{+}$ calculated for $\mathrm{C}_{14} \mathrm{H}_{11} \mathrm{NO}_{5} \mathrm{SNa} 296.0357$, found 296.0349. (Z-isomer, minor): (152.5 mg, 28\%). Yellow oil: ${ }^{1} \mathrm{H}$ NMR $\left(\mathrm{CDCl}_{3}, 500 \mathrm{MHz}\right): \delta=3.69(\mathrm{~s}, 3 \mathrm{H}), 5.07(\mathrm{~d}, 1 \mathrm{H}, J=7.1 \mathrm{~Hz}), 6.16$ $(\mathrm{s}, 1 \mathrm{H}), 6.65(\mathrm{~d}, 1 \mathrm{H}, J=7.1 \mathrm{~Hz}), 7.42-7.48(\mathrm{~m}, 2 \mathrm{H}), 7.86(\mathrm{~s}, 1 \mathrm{H}), 7.88(\mathrm{~d}, 1 \mathrm{H}, J=7.0 \mathrm{~Hz}), 7.95$ $(\mathrm{d}, 1 \mathrm{H}, J=7.0 \mathrm{~Hz}),{ }^{13} \mathrm{C} \mathrm{NMR}\left(\mathrm{CDCl}_{3}, 125 \mathrm{MHz}\right): \delta=51.2,67.2,101.0,115.0,122.0,123.0$, $125.3,125.6,125.9,129.2,135.4,140.7,152.0,164.4$ ppm.

Methyl 3-(1-cyanopropoxy)acrylate (3r). (E-isomer, major): (207.5 mg, 62\%). Transparent liquid: ${ }^{1} \mathrm{H}$ NMR $\left(\mathrm{CDCl}_{3}, 400 \mathrm{MHz}\right): \delta=1.11(\mathrm{t}, 3 \mathrm{H}, J=7.4 \mathrm{~Hz}), 1.97-2.04(\mathrm{~m}, 2 \mathrm{H}), 3.70(\mathrm{~s}$, $3 \mathrm{H}), 4.54(\mathrm{t}, 1 \mathrm{H}, J=6.5 \mathrm{~Hz}), 5.41(\mathrm{~d}, 1 \mathrm{H}, J=12.6 \mathrm{~Hz}), 7.56(\mathrm{~d}, 1 \mathrm{H}, J=12.6 \mathrm{~Hz}) .{ }^{13} \mathrm{C} \mathrm{NMR}$ $\left(\mathrm{CDCl}_{3}, 100 \mathrm{MHz}\right): \delta=8.8,26.5,51.4,69.4,100.3,115.9,158.7,166.8$ ppm. HRMS $\left(\mathrm{ESI}^{+}\right)$: $m / z[\mathrm{M}+\mathrm{Na}]^{+}$calculated for $\mathrm{C}_{8} \mathrm{H}_{11} \mathrm{NO}_{3} \mathrm{Na}$ 192.0637, found 192.0633. (Z-isomer, minor): (99.0 mg, 29\%). Transparent liquid: ${ }^{1} \mathrm{H} \mathrm{NMR}\left(\mathrm{CDCl}_{3}, 400 \mathrm{MHz}\right): \delta=1.13(\mathrm{t}, 3 \mathrm{H}, J=7.4$ $\mathrm{Hz}), 1.99-2.07(\mathrm{~m}, 2 \mathrm{H}), 3.68(\mathrm{~s}, 3 \mathrm{H}), 4.60(\mathrm{t}, 1 \mathrm{H}, J=6.5 \mathrm{~Hz}), 5.07(\mathrm{~d}, 1 \mathrm{H}, J=7.0 \mathrm{~Hz}), 6.58(\mathrm{~d}$, $1 \mathrm{H}, J=7.0 \mathrm{~Hz}) .{ }^{13} \mathrm{C} \mathrm{NMR}\left(\mathrm{CDCl}_{3}, 100 \mathrm{MHz}\right): \delta=8.8,26.7,51.1,72.1,100.1,116.4,153.8$, $164.5 \mathrm{ppm}$.

Methyl 3-(1-cyanobutoxy)acrylate (7s). (E-isomer, major): (219.4 mg, 59\%). Transparent liquid: ${ }^{1} \mathrm{H}$ NMR $\left(\mathrm{CDCl}_{3}, 400 \mathrm{MHz}\right): \delta=0.95(\mathrm{t}, 3 \mathrm{H}, J=7.2 \mathrm{~Hz}), 1.46-1.55(\mathrm{~m}, 2 \mathrm{H}), 1.87-1.94$ $(\mathrm{m}, 2 \mathrm{H}), 3.66(\mathrm{~s}, 3 \mathrm{H}), 4.58(\mathrm{t}, 1 \mathrm{H}, J=6.5 \mathrm{~Hz}), 5.39(\mathrm{~d}, 1 \mathrm{H}, J=12.6 \mathrm{~Hz}), 7.46(\mathrm{~d}, 1 \mathrm{H}, J=12.6 \mathrm{~Hz})$. ${ }^{13} \mathrm{C} \mathrm{NMR}\left(\mathrm{CDCl}_{3}, 100 \mathrm{MHz}\right): \delta=13.1,17.6,34.6,51.2,68.1,100.2,116.1,158.7,166.7 \mathrm{ppm}$. HRMS $\left(\mathrm{ESI}^{+}\right): m / z[\mathrm{M}+\mathrm{Na}]^{+}$calculated for $\mathrm{C}_{9} \mathrm{H}_{13} \mathrm{NO}_{3} \mathrm{Na} 206.0793$, found 206.0792. (Zisomer, minor): (113.6 mg, 31\%). Transparent liquid: ${ }^{1} \mathrm{H} \mathrm{NMR}\left(\mathrm{CDCl}_{3}, 400 \mathrm{MHz}\right): \delta=0.98$ $(\mathrm{t}, 3 \mathrm{H}, J=7.1 \mathrm{~Hz}), 1.54-1.63(\mathrm{~m}, 2 \mathrm{H}), 1.93-2.03(\mathrm{~m}, 2 \mathrm{H}), 3.69(\mathrm{~s}, 3 \mathrm{H}), 4.64(\mathrm{t}, 1 \mathrm{H}, J=6.5 \mathrm{~Hz})$, $5.07(\mathrm{~d}, 1 \mathrm{H}, J=7.1 \mathrm{~Hz}), 6.57(\mathrm{~d}, 1 \mathrm{H}, J=7.1 \mathrm{~Hz}) .{ }^{13} \mathrm{C} \mathrm{NMR}\left(\mathrm{CDCl}_{3}, 100 \mathrm{MHz}\right): \delta=13.2,17.8$, $35.0,51.1,70.9,100.1,116.5,153.8,164$ ppm.

Methyl 3-((1-cyanopentyl)oxy)acrylate (7t). (E-isomer, major): (169.3 mg, 43\%). Transparent oil: ${ }^{1} \mathrm{H} \mathrm{NMR}\left(\mathrm{CDCl}_{3}, 500 \mathrm{MHz}\right): \delta=0.90(\mathrm{t}, 3 \mathrm{H}, J=7.72 \mathrm{~Hz}), 1.31-1.39(\mathrm{~m}, 2 \mathrm{H})$, $1.44-1.50(\mathrm{~m}, 2 \mathrm{H}), 1.92-1.96(\mathrm{~m}, 2 \mathrm{H}), 3.68(\mathrm{~s}, 3 \mathrm{H}), 4.58(\mathrm{t}, 1 \mathrm{H}, J=6.50 \mathrm{~Hz}), 5.40(\mathrm{~d}, 1 \mathrm{H}$, $J=12.8 \mathrm{~Hz}), 7.47(\mathrm{~d}, 1 \mathrm{H}, J=12.8 \mathrm{~Hz}) .{ }^{13} \mathrm{C} \mathrm{NMR}\left(\mathrm{CDCl}_{3}, 125 \mathrm{MHz}\right): \delta=13.5,21.8,26.3$, $32.5,51.3,68.3,100.2,116.1,158.7,166.8 \mathrm{ppm}$. HRMS $\left(\mathrm{ESI}^{+}\right): \mathrm{m} / z[\mathrm{M}+\mathrm{Na}]^{+}$calculated for $\mathrm{C}_{10} \mathrm{H}_{15} \mathrm{NO}_{3} 220.0950$, found 220.0952. (Z-isomer, minor): (88.2 mg, 22\%). Transparent oil: ${ }^{1} \mathrm{H} \mathrm{NMR}\left(\mathrm{CDCl}_{3}, 500 \mathrm{MHz}\right): \delta=0.92(\mathrm{t}, 3 \mathrm{H}, J=7.2 \mathrm{~Hz}), 1.34-1.41(\mathrm{~m}, 2 \mathrm{H}), 1.48-1.54(\mathrm{~m}$, $2 \mathrm{H}), 1.94-2.02(\mathrm{~m}, 2 \mathrm{H}), 3.67(\mathrm{~s}, 3 \mathrm{H}), 4.63(\mathrm{t}, 1 \mathrm{H}, J=6.5 \mathrm{~Hz}), 5.06(\mathrm{~d}, 1 \mathrm{H}, J=7.14 \mathrm{~Hz}), 6.57(\mathrm{~d}$, $1 \mathrm{H}, J=7.1 \mathrm{~Hz}) .{ }^{13} \mathrm{C} \mathrm{NMR}\left(\mathrm{CDCl}_{3}, 125 \mathrm{MHz}\right): \delta=13.6,21.8,26.3,32.7,51.0,71.1,99.9,116.5$, $153.8,164.5 \mathrm{ppm}$.

Methyl 3-((1-cyanoheptyl)oxy)acrylate (7u). (E-isomer, major): (173.3 mg, 39\%). Transparent oil: ${ }^{1} \mathrm{H}$ NMR $\left(\mathrm{CDCl}_{3}, 500 \mathrm{MHz}\right): \delta=0.84(\mathrm{t}, 3 \mathrm{H}, J=6.9 \mathrm{~Hz}), 1.25-1.33(\mathrm{~m}, 6 \mathrm{H}), 1.43-1.49$ $(\mathrm{m}, 2 \mathrm{H}), 1.90-1.95(\mathrm{~m}, 2 \mathrm{H}), 3.66(\mathrm{~s}, 3 \mathrm{H}), 4.58(\mathrm{t}, 1 \mathrm{H}, J=6.5 \mathrm{~Hz}), 5.39(\mathrm{~d}, 1 \mathrm{H}, J=12.2 \mathrm{~Hz})$, $7.47(\mathrm{~d}, 1 \mathrm{H}, J=12.2 \mathrm{~Hz}) .{ }^{13} \mathrm{C} \mathrm{NMR}\left(\mathrm{CDCl}_{3}, 125 \mathrm{MHz}\right): \delta=13.7,22.2,24.1,28.2,31.2,32.7$, 51.2, 68.3, 100.1, 116.0, 158.6, $166.7 \mathrm{ppm}$. HRMS $\left(\mathrm{ESI}^{+}\right): \mathrm{m} / z[\mathrm{M}+\mathrm{Na}]^{+}$calculated for $\mathrm{C}_{12} \mathrm{H}_{19} \mathrm{NO}_{3} \mathrm{Na} 248.1263$, found 248.1260. (Z-isomer, minor): (73.0 mg, 16\%). Transparent oil: ${ }^{1} \mathrm{H} \mathrm{NMR}\left(\mathrm{CDCl}_{3}, 500 \mathrm{MHz}\right): \delta=0.87(\mathrm{t}, 3 \mathrm{H}, J=6.9 \mathrm{~Hz}), 1.27-1.31(\mathrm{~m}, 4 \mathrm{H}), 1.32 .-1.38$ $(\mathrm{m}, 2 \mathrm{H}), 1.50-1.56(\mathrm{~m}, 2 \mathrm{H}), 1.95-2.03(\mathrm{~m}, 2 \mathrm{H}), 3.68(\mathrm{~s}, 3 \mathrm{H}), 4.64(\mathrm{t}, 1 \mathrm{H}, J=6.8 \mathrm{~Hz}), 5.06(\mathrm{~d}$, $1 \mathrm{H}, J=7.1 \mathrm{~Hz}), 6.58(\mathrm{~d}, 1 \mathrm{H}, J=7.1 \mathrm{~Hz}) .{ }^{13} \mathrm{C} \mathrm{NMR}\left(\mathrm{CDCl}_{3}, 125 \mathrm{MHz}\right): \delta=13.9,22.4,24.3$, 28.3, 31.3, 33.0, 51.1, 71.1, 100.1, 153.8, 164.5 ppm.

Methyl 3-(1-cyano-2-methylpropoxy)acrylate (7v). (E-isomer, major): (181.7 mg, 50\%). Transparent oil: ${ }^{1} \mathrm{H}$ NMR $\left(\mathrm{CDCl}_{3}, 400 \mathrm{MHz}\right): \delta=1.05(\mathrm{~d}, 3 \mathrm{H}, J=6.7 \mathrm{~Hz}), 1.08(\mathrm{~d}, 3 \mathrm{H}$, $J=6.7 \mathrm{~Hz}), 2.15-2.21(\mathrm{~m}, 1 \mathrm{H}), 3.66(\mathrm{~s}, 3 \mathrm{H}), 4.40(\mathrm{~d}, 1 \mathrm{H}, J=5.8 \mathrm{~Hz}), 5.46(\mathrm{~d}, 1 \mathrm{H}, J=12.7 \mathrm{~Hz})$, $7.47(\mathrm{~d}, 1 \mathrm{H}, \mathrm{J}=12.7 \mathrm{~Hz}) .{ }^{13} \mathrm{C} \mathrm{NMR}\left(\mathrm{CDCl}_{3}, 100 \mathrm{MHz}\right): \delta=17.0,17.4,51.2,73.7,100.0,115.2$, 158.9, 166.8 ppm. HRMS $\left(\mathrm{ESI}^{+}\right): m / z[\mathrm{M}+\mathrm{Na}]^{+}$calculated for $\mathrm{C}_{9} \mathrm{H}_{13} \mathrm{NO}_{3} \mathrm{Na} 206.0793$, found 206.0792. (Z-isomer, minor): (85.5 mg, 23\%). Yellow oil: ${ }^{1} \mathrm{H} \mathrm{NMR}\left(\mathrm{CDCl}_{3}, 400 \mathrm{MHz}\right)$ : 
$\delta=1.11(\mathrm{~d}, 3 \mathrm{H}, J=6.7 \mathrm{~Hz}), 1.14(\mathrm{~d}, 3 \mathrm{H}, J=6.7 \mathrm{~Hz}), 2.20-2.29(\mathrm{~m}, 1 \mathrm{H}), 3.68(\mathrm{~s}, 3 \mathrm{H}), 4.39(\mathrm{~d}$, $1 \mathrm{H}, J=6.9 \mathrm{~Hz}), 5.05(\mathrm{~d}, 1 \mathrm{H}, J=7.0 \mathrm{~Hz}), 6.56(\mathrm{~d}, 1 \mathrm{H}, J=7.0 \mathrm{~Hz}) .{ }^{13} \mathrm{C} \mathrm{NMR}\left(\mathrm{CDCl}_{3}, 100 \mathrm{MHz}\right)$ : $\delta=17.3,17.6,51.1,76.7,100.0,115.7,154.1,164.6$ ppm.

Methyl 3-(1-cyano-2,2-dimethylpropoxy)acrylate (7w). (E-isomer, major): (534 mg, 68\%). Transparent oil: ${ }^{1} \mathrm{H} \mathrm{NMR}\left(\mathrm{CDCl}_{3}, 500 \mathrm{MHz}\right): \delta=1.11(\mathrm{~s}, 9 \mathrm{H}), 3.71(\mathrm{~s}, 3 \mathrm{H}), 4.19(\mathrm{~s}, 1 \mathrm{H}), 5.44$ $(\mathrm{d}, 1 \mathrm{H}, J=12.7 \mathrm{~Hz}), 7.52(\mathrm{~d}, 1 \mathrm{H}, J=12.7 \mathrm{~Hz}) .{ }^{13} \mathrm{C}$ NMR $\left(\mathrm{CDCl}_{3}, 125 \mathrm{MHz}\right): \delta=25.0(3 \mathrm{C})$, 35.4, 51.4, 77.5, 100.0, 115.2, 159.2, 166.9 ppm. HRMS $\left(\mathrm{ESI}^{+}\right): \mathrm{m} / z[\mathrm{M}+\mathrm{Na}]^{+}$calculated for $\mathrm{C}_{10} \mathrm{H}_{15} \mathrm{NO}_{3} \mathrm{Na} 220.0950$, found 220.0951. (Z-isomer, minor): (213.5 $\left.\mathrm{mg}, 27 \%\right)$. White solid: ${ }^{1} \mathrm{H}$ NMR $\left(\mathrm{CDCl}_{3}, 500 \mathrm{MHz}\right): \delta=1.11(\mathrm{~s}, 9 \mathrm{H}), 3.66(\mathrm{~s}, 3 \mathrm{H}), 4.21(\mathrm{~s}, 1 \mathrm{H}), 5.01(\mathrm{~d}, 1 \mathrm{H}$, $J=6.8 \mathrm{~Hz}), 6.56(\mathrm{~d}, 1 \mathrm{H}, J=6.8 \mathrm{~Hz}) .{ }^{13} \mathrm{C} \mathrm{NMR}\left(\mathrm{CDCl}_{3}, 125 \mathrm{MHz}\right): \delta=25.0(3 \mathrm{C}), 35.6,50.9$, $80.3,99.5,115.7,154.8,164.5$ ppm.

\section{Conclusions}

We have shown that the organocatalytic multicomponent cyanovinylation of aldehydes is a powerful synthetic tool for the synthesis of conjugated cyanomethyl vinyl ethers 3. The power of this synthetic tool was demonstrated in the synthesis of an array of 3(cyanomethoxy)acrylates 7 endowed with a diverse substitution pattern at the position C-3. The reaction was performed in a three-component format using as inputs an aldehyde, acetone cyanohydrin (5), methyl propiolate (4) and a catalytic amount of $N$-methylmorpholine. The 3CR was performed at room temperature in $n$-hexanes to deliver $(E / Z)$-3-substituted3-(cyanomethoxy)acrylates 7 in excellent yields and with a preponderance of the $E$-isomer. The reaction is highly tolerant to the structure and composition of the aldehyde (aliphatic, aromatic, heteroaromatic), and it is instrumentally simple (one batch, open atmospheres), economical ( $2.5 \mathrm{~mol} \%$ catalyst, stoichiometric reagents), environmentally friendly (no toxic waste), and sustainable (easy scalability).

Supplementary Materials: ${ }^{1} \mathrm{H}$ and ${ }^{13} \mathrm{C}$ NMR spectra for compounds 7 are available online.

Author Contributions: S.D.-H. conducted experimental work; D.T. and F.G.-T. initiated the project, designed experiments and wrote the paper. All authors have read and agreed to the published version of the manuscript.

Funding: This research was funded by the Spanish Ministry of Science, Innovation and Universities (MICINN), State Research Agency (AEI) and the European Regional Development Funds (ERDF) (PGC2018-094503-B-C21).

Acknowledgments: S.D.H. thanks La Laguna University and Cajasiete for a pre-doctoral contract, and Ministerio de Ciencia, Innovación y Universidades.

Conflicts of Interest: The authors declare no conflict of interest.

Sample Availability: Samples of the compounds 7a-w are available from the authors.

\section{References and Note}

1. Sugihara, S. Alkenyl Ether-Vinyl Ester Copolymer. WO 2016181873, 17 November 2016.

2. Kim, K.-Y.; Kim, N.-M.; Yoon, S.-S. Separator, and Electrochemical Device Comprising Same. WO 2014054919,10 April 2014.

3. Kim, H.S. Separator and Electrochemical Device Containing Thereof. Repub. KR 2017095024, 22 August 2017.

4. Kim, G.Y.; Yoon, S.S.; Kim, N.M.; Yoon, J.A.; Hwang, I.H.; Kim, S.J. Binder Resin Composition, Porous Separator Comprising It, Electrochemical Element Using It. Repub. KR 2014147742, 30 December 2014.

5. Holder, A.J.; Morrill, J.A.; White, D.A.; Eick, J.D.; Chappelow, C.C. A semiempirical quantum mechanical study of cationically catalyzed homopolymerization and copolymerization of vinyl ethers and epoxides. J. Mol. Struct. 2000, 507, 63-73. [CrossRef]

6. Sun, Z.; Li, Z.; Liao, W.-W. An organocatalytic hydroalkoxylation/Claisen rearrangement/Michael addition tandem sequence: Divergent synthesis of multi-substituted 2,3-dihydrofurans and 2,3-dihydropyrroles from cyanohydrins. Green Chem. 2019, 21, 1614-1618. [CrossRef]

7. Du, S.-T.; Sun, Z.; Liu, W.; Liao, W.-W. Lewis Base-Catalyzed One-Pot Cascade Sequences of O-Alkenyl-Substituted Cyanohydrins: Diastereoselective Synthesis of Multisubstituted Dihydrofurans. Org. Lett. 2017, 19, 6598-6601. [CrossRef] [PubMed]

8. Aviyente, V.; Houk, K.N. Cyano, Amino, and Trifluoromethyl Substituent Effects on the Claise6n Rearrangement. J. Phys. Chem. A 2001, 105, 383-391. 
9. Burrows, C.J.; Carpenter, B.K. Substituent Effects on the Aliphatic Claisen Rearrangement. 1. Synthesis and Rearrangement of Cyano-Substituted Allyl Vinyl Ethers. J. Am. Chem. Soc. 1981, 103, 6983-6984.

10. Dewar, M.J.S.; Caoxian, J. Mechanism of the Claisen Rearrangement of Allyl Vinyl Ethers. J. Am. Chem. Soc. 1989, 111, 511-519. [CrossRef]

11. We have not found precedents for this transformation. Searchs in Scifinder under reaction substructure, structure or research topic did not show results for this transformation.

12. Tejedor, D.; Delgado-Hernández, S.; Colella, L.; García-Tellado, F. Catalytic Hydrocyanation of Activated Terminal Alkynes. Chem. Eur. J. 2019, 25, 15046-15049. [CrossRef] [PubMed]

13. Tejedor, D.; López-Tosco, S.; Cruz-Acosta, F.; Méndez-Abt, G.; García-Tellado, F. Acetylides from Alkyl Propiolates as Building Blocks for C3 Homologation. Angew. Chem. Int. Ed. 2009, 48, 2090-2098. [CrossRef] [PubMed]

14. Tejedor, D.; García-Tellado, F.; Marrero-Tellado, J.J.; de Armas, P. Efficient Domino Process Based on the Catalytic Generation of Non-Metalated, Conjugated Acetylides in the Presence of Aldehydes or Activated Ketones. Chem. Eur. J. 2003, 9, $3122-3131$. [CrossRef] [PubMed]

15. Stewart, I.C.; Toste, F.D.; Bergman, R.G. Phosphine-Catalyzed Hydration and Hydroalkoxylation of Activated Olefins: Use of a Strong Nucleophile to Generate a Strong Base. J. Am. Chem. Soc. 2003, 125, 8696-8697. [CrossRef] [PubMed]

16. de Armas, P.; García-Tellado, F.; Marrero-Tellado, J.J.; Tejedor, D.; Maestro, M.A.; González-Platas, J. Alkynoates as a Source of Reactive Alkylinides for Aldehyde Addition Reactions. Org. Lett. 2001, 3, 1905-1908. [CrossRef] [PubMed]

17. Haroutounian, S.A. Acetone Cyanohydrin. In Encyclopedia of Reagents for Organic Synthesis; John Wiley \& Sons: New York, NY, USA, 2001.

18. Nauth, A.M.; Opatz, T. Non-toxic cyanide sources and cyanating agents. Org. Biomol. Chem. 2019, 17, 11-23. [CrossRef] [PubMed]

19. Tejedor, D.; Álvarez-Méndez, S.J.; López-Soria, J.M.; Martín, V.S.; García-Tellado, F. A robust and general protocol for the Lewis-base-catalysed reaction of alcohols and alkyl propiolates. Eur. J. Org. Chem. 2014, 2014, 198-205. [CrossRef] 